This is the accepted manuscript made available via CHORUS. The article has been published as:

\title{
Shapes of rotating superfluid helium nanodroplets
}

Charles Bernando, Rico Mayro P. Tanyag, Curtis Jones, Camila Bacellar, Maximilian Bucher, Ken R. Ferguson, Daniela Rupp, Michael P. Ziemkiewicz, Luis F. Gomez, Adam S. Chatterley, Tais Gorkhover, Maria Müller, John Bozek, Sebastian Carron, Justin Kwok, Samuel L. Butler, Thomas Möller, Christoph Bostedt, Oliver Gessner, and Andrey F. Vilesov

Phys. Rev. B 95, 064510 - Published 16 February 2017

DOI: 10.1103/PhysRevB.95.064510 


\section{Shapes of Rotating Superfluid Helium Nano-droplets}

Charles Bernando1, Rico Mayro P. Tanyag 2 , Curtis Jones ${ }^{2}$, Camila Bacellar, ${ }^{3,4}$, Maximilian Bucher $^{5,11}$, Ken R. Ferguson ${ }^{5,6}$, Daniela Rupp ${ }^{7}$, Michael Ziemkiewicz ${ }^{3,4,8}$, Luis F. Gomez, ${ }^{2, *}$, Adam S. Chatterley ${ }^{3, \$}$, Tais Gorkhover ${ }^{5,7}$, Maria Mueller ${ }^{7}$, John Bozek $^{5}$, Sebastian Carron ${ }^{5, \dagger}$,

Justin Kwok ${ }^{8, \pi}$, Samuel L. Butler ${ }^{9}$, Thomas Moeller ${ }^{7}$, Christoph Bostedt ${ }^{5,10,11,12, a)}$, Oliver Gessner $^{3, \text { a }}$ and Andrey F. Vilesov ${ }^{1,2, a)}$

${ }^{1}$ Department of Physics and Astronomy, University of Southern California, Los Angeles, California 90089, USA

${ }^{2}$ Department of Chemistry, University of Southern California, Los Angeles, California 90089, USA

${ }^{3}$ Chemical Sciences Division, Lawrence Berkeley National Laboratory, Berkeley, California 94720, USA

${ }^{4}$ Department of Chemistry, University of California Berkeley, Berkeley, California 94720, USA

${ }^{5}$ Linac Coherent Light Source, LCLS, SLAC National Accelerator Laboratory, 2575 Sand Hill Road, Menlo Park, California 94025, USA

${ }^{6}$ Department of Applied Physics, Stanford University, Stanford, California 94305, USA

${ }^{7}$ Institute of Optics and Atomic Physics, Technical University of Berlin, Hardenbergstraße 36, 10623 Berlin, Germany

${ }^{8}$ Mork Family Department of Chemical Engineering and Materials Science, USC, Los Angeles, California 90089, USA

${ }^{9}$ Department of Geological Sciences, University of Saskatchewan, Saskatoon, SK S7N5E2, Canada

${ }^{10}$ PULSE Institute, Stanford University and SLAC National Accelerator Laboratory, 2575 Sand Hill Road, Menlo Park, California 94025, USA

${ }^{11}$ Argonne National Laboratory, 9700 South Cass Avenue B109, Lemont, Illinois 60439, USA

12 Department of Physics and Astronomy, Northwestern University, 2145 Sheridan Road, Evanston, Illinois 60208, USA

$\S$ Present address: Vescent Photonics, Inc., 14998 W. $6^{\text {th }}$ Ave. Suite 700, Golden, Colorado 80401, USA

* Present address: IPG Photonics, 3930 Freedom Circle, Ste. 130, Santa Clara, California 95054, USA

† Present address: Department of Chemistry, Aarhus University, Langelandsgade 140, 8000 Aarhus C, Denmark

$\dagger$ Present address: California Lutheran University, 60 Olsen Rd, Thousand Oaks, California 91360, USA

I Present address: Department of Materials Science and Engineering, University of Illinois at UrbanaChampaign, Urbana, Illinois 61801, USA

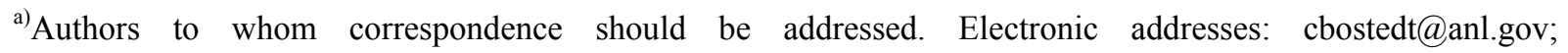
ogessner@lbl.gov; and vilesov@usc.edu. 


\begin{abstract}
Rotating superfluid He droplets of approximately $1 \mu \mathrm{m}$ in diameter were obtained in a free nozzle beam expansion of liquid $\mathrm{He}$ in vacuum and were studied by single-shot coherent diffractive imaging using an x-ray free electron laser. The formation of strongly deformed droplets is evidenced by large anisotropies and intensity anomalies (streaks) in the obtained diffraction images. The analysis of the images shows that, in addition to previously described axially symmetric oblate shapes, some droplets exhibit prolate shapes. Forward modeling of the diffraction images indicates that the shapes of rotating superfluid droplets are very similar to their classical counterparts, giving direct access to the droplet angular momenta and angular velocities. The analyses of the radial intensity distribution and appearance statistics of the anisotropic images confirm the existence of oblate metastable superfluid droplets with large angular momenta beyond the classical bifurcation threshold.
\end{abstract}




\section{Introduction}

Starting with Newton, the equilibrium shapes of rotating classical bodies held together by gravitation have attracted great interest [1]. It has been shown that the shapes of rotating liquid droplets held together by capillary forces belong to the same class of solutions and can serve as laboratory scale emulations of astronomical objects [2]. Liquid drop models have also been applied to predict the shapes of rotating atomic nuclei [3]. Equilibrium shapes of classical rotating droplets have been extensively studied theoretically [2, 4-7] and experimentally [7-8]. A droplet that is spherical at rest acquires an oblate axially symmetric shape upon rotation and, with increasing angular momentum, turns into a two-lobed figure that is elongated perpendicular to the rotational axis, as discussed in more detail in Section 4. This paper extends the study of equilibrium shapes of rotating liquids from classical, viscous droplets to viscosity-free, superfluid droplets. Classical rigid body rotation (RBR) is not feasible in a superfluid droplet, which instead rotates through the emergence of a collection of quantum vortices [9-12]. Each vortex is characterized by a quantized velocity circulation $\kappa=h / M$, which is the ratio of Planck's constant, $h$, and the mass of the ${ }^{4} \mathrm{He}$ atom, $M$. Thus, the velocity field in a superfluid droplet deviates considerably from that in RBR, which may have an impact on the droplet shape and stability. Furthermore, viscous energy dissipation in classical droplets facilitates shape transformations whereby the total angular momentum is conserved but the total kinetic energy is reduced. Negligible viscosity, therefore, may cause some unique shapes of superfluid droplets, which are of particular interest. Vortices in helium droplets have been considered theoretically [13-15] and were searched for experimentally by levitating superfluid ${ }^{4} \mathrm{He}$ droplets in inhomogeneous magnetic fields [16]. It was also observed that charged droplets in a rotating 
electric field develop pronounced deformations that were ascribed to the excitation of capillary waves travelling along the droplet's equator [17].

Very recently we have shown that swiftly rotating ${ }^{4} \mathrm{He}$ droplets of sub-micrometer size may be produced from a cryogenic jet expansion into vacuum and that the droplet shapes can be studied via scattering of radiation from an x-ray free-electron laser (XFEL) [18]. X-ray diffraction imaging of Xe doped He droplets revealed Bragg spots, confirming the existence of quantum vortex lattices that led to the condensation of 100-200 Xe clusters in a periodic array [18]. Positions and shapes of individual vortices could be deduced from diffraction images without Bragg spots by using a recently developed phase retrieval algorithm [19-20]. It was also found that about $50 \%$ of the droplets produce anisotropic diffraction patterns that can be described by concentric elliptic rings. Such elliptic diffraction patterns were ascribed to pseudospheroidal shapes of rotating droplets with aspect ratios (AR) up to $A R=1.5$. In addition, approximately $1 \%$ of the diffraction images exhibit streaks, i.e., pronounced intensity anomalies radiating away from the image center, see Fig. 1 (f-j) [18]. These images cannot be described by elliptical diffraction contours and exhibit very high aspect ratios of $1.5<\mathrm{AR}<2.3$. In Ref. [18] we assigned such images to superfluid droplets that remain axially symmetric beyond their classical stability range, in agreement with the predicted extended range of stability in rotating inviscid droplets [4] and recent DFT calculations [21]. However, the question remained whether prolate superfluid droplets may exist as well for a sufficiently high degree of rotational excitation. Here, we report on a new set of single-shot $\mathrm{x}$-ray coherent diffractive imaging experiments to clarify these questions. The existence of prolate droplet shapes is unambiguously demonstrated by the observation of diffraction images with curved streaks, and the appearance of streaks in images with small aspect ratios. A Fourier Transform based reconstruction of the 
droplets' contours reveals the existence of pronounced dimples, characteristic for prolate droplet shapes at high angular momenta. On the other hand, the formation of oblate helium nanodroplets beyond the stability range of their classical counterparts was confirmed by diffraction images with elliptical contours having aspect ratio $>1.5$. The abundance statistics of different classes of strongly deformed droplets in the beam is in agreement with the presence of both strongly deformed oblate as well as prolate droplets in the beam. The diffraction images can be well described by employing equilibrium shapes of either axially symmetric oblate or triaxial prolate classical droplets. It follows that the shapes of rotating sub-micron superfluid droplets are very similar to those of their classical counterparts, giving access to the droplets' angular momenta and angular velocities.

\section{Experimental}

The experiments were performed at the Atomic, Molecular and Optical Sciences beamline (AMO) of the Linac Coherent Light Source (LCLS). In contrast to our previous experiments (run 1), [18] which employed radiation with $\lambda=0.826 \mathrm{~nm}(\mathrm{~h} v=1.5 \mathrm{KeV})$, the latest experiments (run 2) were performed at $\lambda=1.46 \mathrm{~nm}(\mathrm{~h} v=850 \mathrm{eV})$. The $\mathrm{x}$-ray beam in experimental run 2 also had a smaller nominal focus size of $\approx 2 \mu \mathrm{m}$ as compared to $\approx 5 \mu \mathrm{m}$ in experimental run 1 [18]. As a result, diffraction signals at larger scattering angles could be detected, revealing additional information on the three-dimensional shapes of the droplets. These latest experiments utilized the new "LAMP" soft x-ray end station [22-23]. Experiments with beams of helium nanodroplets have been extensively documented elsewhere [24-27]. Helium droplets with radii $R=300-1000 \mathrm{~nm}\left(\mathrm{~N}_{\mathrm{He}}=10^{9}-10^{11}\right)$ were produced upon fragmentation of liquid ${ }^{4} \mathrm{He}$ expanding continuously into vacuum through a $5 \mu \mathrm{m}$ diameter nozzle at a temperature

of $5 \mathrm{~K}$ and a backing pressure of 20 bar [24, 28-29]. The droplets rapidly cool down via 
evaporation and became superfluid at $\mathrm{T}<2.17 \mathrm{~K}$. As previously observed [18], the droplets have considerable angular momentum, which likely originates from inhomogeneous flow of helium through the nozzle during the expansion [30]. After travelling across a distance of $\approx 700$ $\mathrm{mm}$ from the nozzle within approximately $4 \mathrm{~ms}$, the droplets traversed the focus of the XFEL beam (pulse energy $\mathrm{E}_{\text {pulse }} \approx 0.7 \mathrm{~mJ}$, repetition rate $120 \mathrm{~Hz}$ ). The duration of the x-ray laser pulses $(60 \mathrm{fs})$ is much shorter than the typical droplet rotational period (> $100 \mathrm{~ns})$ and the probability to find a droplet within the tight focus of the x-ray beam at any given time is less than $10^{-3}$. Thus, every diffraction image represents the instantaneous configuration of a single helium droplet. The images were recorded using two pn-CCD detectors comprising about one million $75 \times 75$ $\mu \mathrm{m}^{2}$ pixels each that were placed at distances of $\approx 370 \mathrm{~mm}$ (front) and $\approx 735 \mathrm{~mm}$ (rear) from the interaction center [31-33]. Each detector consisted of two $\left(75 \times 37.5 \mathrm{~mm}^{2}\right) \mathrm{CCD}$ panels that were separated by $32.8 \mathrm{~mm}$ and $1.5 \mathrm{~mm}$ gaps for the front and rear detectors, respectively, to let the primary $\mathrm{x}$-ray beam pass.

\section{Results}

Figure 1 shows some characteristic diffraction images from He droplets obtained with the rear pnCCD detector, which are displayed in a logarithmic scale. The images are analyzed in terms of the aspect ratio of the diffraction contours, AR, which is the inverse of the aspect ratio of the droplet's projections onto the detector plane, see Ref. [18] and its supplementary materials (SM). The values for the AR as well as the corresponding major and minor semi-axis of the droplet's projections obtained from the images in Fig. 1 are summarized in Table 1. The complete collection of images discussed in this work and the results of the corresponding data analysis are presented in section S1 in SM. Fig. 1 a) shows an image with noticeable ellipticity of the diffraction contours, corresponding to an aspect ratio of $\mathrm{AR}=1.105$. 

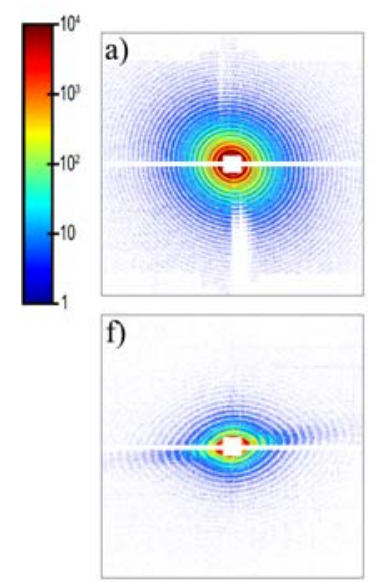

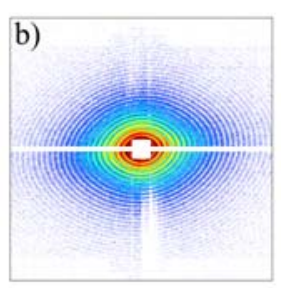

g)

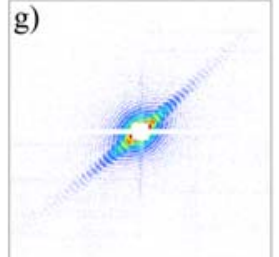

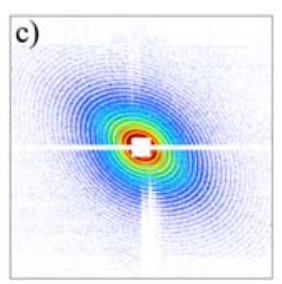

h)

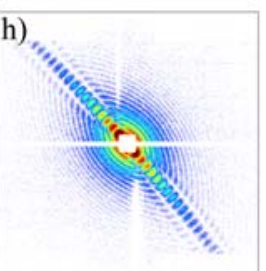

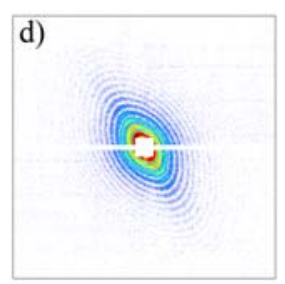

i)

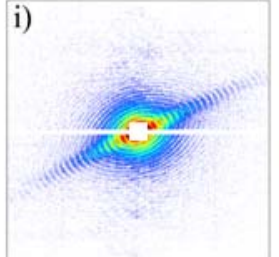

e)

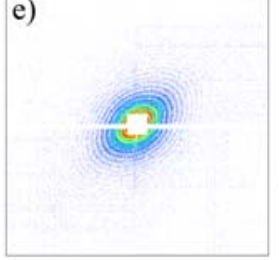

j)

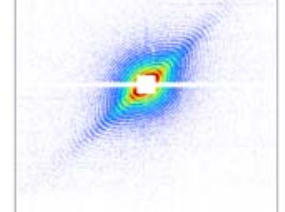

Fig. 1. Diffraction images from He droplets obtained with the rear pn-CCD $(1024 \times 1024$ pixels $)$ detector. The logarithmic intensity color scale is shown on the left. Images (f-j) in the bottom row contain streaks, whereas images (a-e) in the upper row exemplify patterns with large aspect ratios devoid of any streaks. The blank horizontal stripe results from the gap between the upper and lower plates of the pn-CCD detector. Both pn-CCD plates have a rectangular cut next to the gap to accommodate the primary x-ray beam. The vertical stripes in a), b), c) and h) are caused by imperfect data readout for strong diffraction images. The empty regions near the top and bottom edges of the images, which are especially noticeable in panels a), b), and c), are the shadows of the front pn-CCD plates. All images were obtained from bare He droplets except in panel i), which was obtained upon doping with $\mathrm{Xe}$ atoms as described in the text.

Panels b)-e) show strongly distorted images with AR in the range of 1.3 to 1.6 . Fig. $1 \mathrm{f}$ ) -j) show even stronger distorted diffraction images with AR in the range of 1.6 to 1.9. These images also reveal some pronounced streaks, i.e., regions of high intensity along the direction of the long axis in the diffraction image, which extend well beyond the continuous diffraction contours. Large $\mathrm{AR}$ and streaks in the diffraction images indicate some pronounced deformations of the droplets, which are the focus of the present paper. Panels $f$ ) - h) and j) show all four streaked events, from a total of 447 images obtained from bare helium droplets during experimental run 2 . This $\approx 1 \%$ abundance of streaked images has also been observed during experimental run 1 [18]. The number of images obtained from experimental run 2 is smaller than the one obtained from run 1 due to a shorter acquisition time. Panel i) shows a streaked event for Xe doped droplets. 
Scattering contributions from the Xe content lead to additional substructures in the diffraction pattern as can be seen in the middle of the upper and lower half-images.

While differently shaped droplets may exhibit very similar 2D projections in the detector plane, the probability to observe, for example, a specific aspect ratio (AR) of the projection can vary significantly. Therefore, we analyze the statistical distribution of ARs for different classes of diffraction images as illustrated in Fig. 2. Fig. 2 shows a histogram of the abundance vs AR for streaked diffraction contours of experimental runs 1 and 2 (red bars). It also contains the frequency count for all images with AR $>1.3$ that do not exhibit well defined streaks, such as in Fig. 1 a)-e) (blue bars). In the following, these data will be referred to as "large AR events".

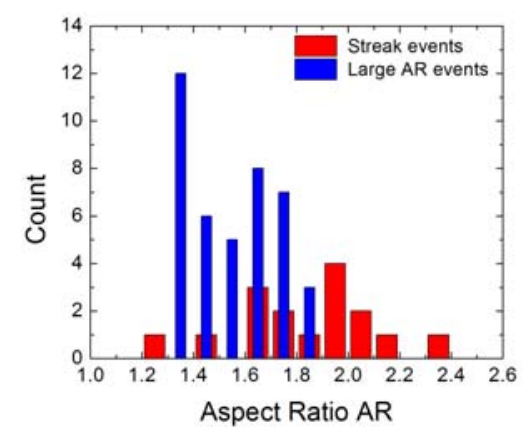

Fig. 2. Abundance of streaked (red) and large AR (blue) events as a function of their aspect ratios. The data includes measurements with bare droplets as well as weakly Xe-doped droplets $\left(\mathrm{N}_{\mathrm{He}} / \mathrm{N}_{\mathrm{Xe}}>1500\right)$. In total, 3140 diffraction images of bare and Xe-doped He droplets were obtained during the two experimental runs.

About $98 \%$ of the obtained images have $1<\mathrm{AR}<1.3$. The data in Figs. $2-4$ contain the results of all relevant measurements from experimental runs 1 and 2 for which aspect ratios could be determined. It is seen that the streaked images concentrate in the range $1.6<\mathrm{AR}<2.1$ and the largest observed aspect ratio is $\mathrm{AR}=2.39$. Only two streaked images were obtained with $\mathrm{AR}<$ 1.6. In contrast, the probability of large AR events devoid of any streaks is distributed almost homogeneously across a narrower range of $1.35 \leq \mathrm{AR} \leq 1.85$. The different abundance distributions 
of streaked and large AR events indicate that the strongly deformed helium droplets in the beam belong to two distinct classes of shapes.

This conclusion is further supported by the detailed analysis of the intensity distributions within the scattering patterns. The appearance of a streak along a particular direction indicates that the scattering object may not be described by a spheroid. For a fixed azimuthal angle of a diffraction image, the scattering intensity scales as the negative power, $\alpha$, of the scattering angle, $\theta$, i.e., $I \propto \theta^{-\alpha}$, see section S2 of the SM. Along azimuthal angles away from the long axes of the images, the fits described in section S2 consistently gave $\alpha \approx 4$ as expected for a spheroid or an ellipsoid. However, along the long axis of the diffraction images, exponents $\alpha$ in the range of $3-4$ were found, signifying a considerable deviation of the droplets from ellipsoidal shapes as will be discussed in the following.

Fig. 3 shows the values of $\alpha$ along the long axis of the images analyzed in Fig. 2. Data points from images with visible streaks are shown as red circles, whereas the data points from large AR events are shown as blue circles. Open circles signify He droplets with a low level of Xe doping, while solid circles correspond to pure droplets. Evidently, data points from bare and slightly Xe doped droplets follow the same trends within the scatter of the data. The large AR events concentrate in the upper part of Fig. 3, and vary across a range of $\alpha=4 \pm 0.2$. On the other hand, the streaked events are concentrated more toward the center of Fig. 3 with $\alpha=3.2$ 3.7. These different distributions again indicate the existence of the two different classes of shapes, such as oblate and prolate, as it will be further discussed in Section 5.1. 


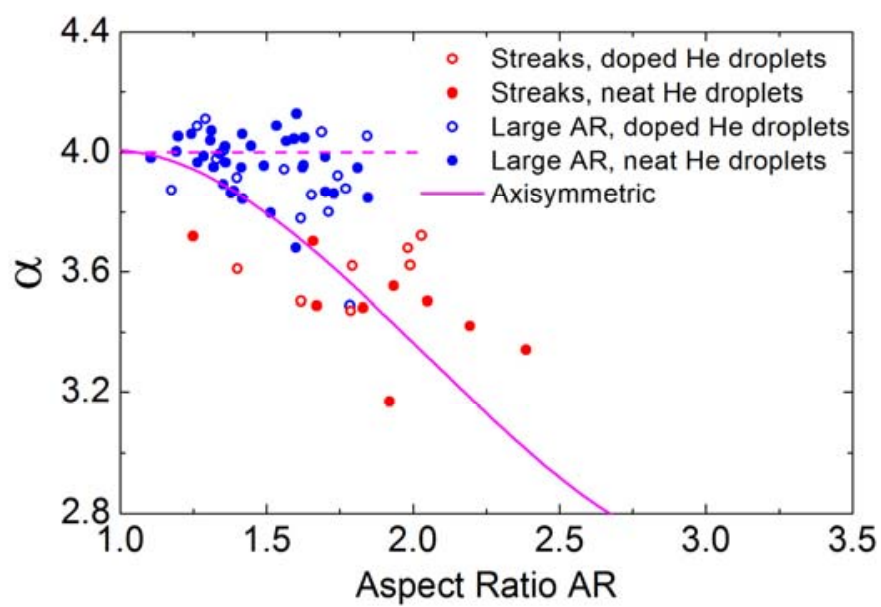

Fig. 3. Correlation between the decay exponent $\alpha$ of the q-dependent scattering intensity and the AR for streaked (red) and large AR images (blue). The solid pink curve corresponds to the expected trend for axisymmetric classical rotating droplets imaged edge on. The data points measured with weakly Xe doped droplets are marked by open circles.

Fig. 4 shows the correlation between semi-major axis, $b$, and semi-minor axis, $a$, for streaked images with discernible diffraction rings. It can be seen that streaks are associated with droplets with semi-minor and semi-major axes in the range of $200-400 \mathrm{~nm}$ and $400-700 \mathrm{~nm}$, respectively. The average aspect ratio is $\langle\mathrm{AR}\rangle=1.86$ as indicated by a linear fit to the data (red line). This rather tight concentration of sizes and aspect ratios of strongly deformed droplets in the beam remains to be explained. In comparison, an ample amount of weakly deformed droplets with average radii in the range of $\mathrm{R}=100-300 \mathrm{~nm}$ and $\mathrm{R}=500-1500 \mathrm{~nm}$ has been observed (not shown). Additionally, five images with streaks (see section S1 in SM) were observed for droplets with semi-minor axes beyond $1000 \mathrm{~nm}$. However the semi-major axes of these droplets could not be determined, since the distance between diffraction contours becomes comparable to the detector pixel size toward the corresponding azimuthal angles. 


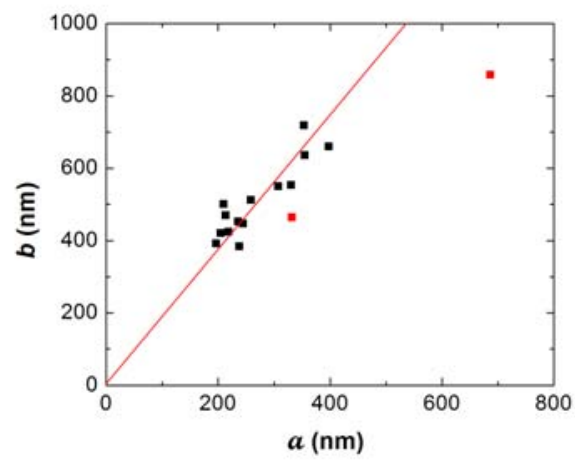

Fig. 4. Correlation between semi-major - $b$ and semi-minor $-a$ axis for images with streaks. The solid line represents a linear fit to the data points, $b=\langle\mathrm{AR}\rangle \cdot a$, giving $\langle\mathrm{AR}\rangle=1.86 \pm 0.05$. The data points in red correspond to $\mathrm{AR}<1.6$, and are not included in the linear fit calculation.

Table 1. Parameters obtained from the diffraction images in Fig. 1: $a$ and $b$ are the semi-minor and semimajor axes, respectively, of the droplet projections onto the diffraction plane; $A R=b / a, \alpha$ is the power of the intensity dependence vs scattering angle along the streaks; $\theta_{\mathrm{MAX}}$ is the maximum scattering angle at which a discernible scattering signal was recorded. The total number of the recorded photons is also listed.

\begin{tabular}{|c|c|c|c|c|c|c|}
\hline $\begin{array}{c}\text { Image } \\
\text { in Fig. } 1 \\
\text { Run number } \\
\text { as in SM }\end{array}$ & $b(\mathrm{~nm})$ & $a(\mathrm{~nm})$ & AR & $\alpha$ & $\theta_{\mathrm{MAX}}(\mathrm{rad})$ & $\begin{array}{c}\text { Number of } \\
\text { photons }\end{array}$ \\
\hline (a) 1623392 & 419.6 & 379.6 & 1.105 & 3.982 & 0.032 & $4.661 \times 10^{6}$ \\
\hline (b) 1551536 & 471.6 & 351.9 & 1.340 & 3.985 & 0.038 & $4.314 \times 10^{6}$ \\
\hline (c) 1603152 & 503.8 & 372.6 & 1.352 & 3.849 & 0.034 & $3.081 \times 10^{6}$ \\
\hline (d) 1603680 & 395.6 & 243.8 & 1.623 & 3.909 & 0.027 & $4.761 \times 10^{5}$ \\
\hline (e) 1577728 & 469.3 & 331.2 & 1.417 & 4.062 & 0.020 & $3.134 \times 10^{5}$ \\
\hline (f) 1573312 & 366 & 229 & 1.597 & 3.662 & 0.039 & $8.453 \times 10^{5}$ \\
\hline (g) 1624432 & 553 & 331 & 1.671 & 3.470 & 0.030 & $3.172 \times 10^{5}$ \\
\hline (h) 1582464 & 452 & 236 & 1.920 & 3.192 & 0.129 & $2.07 \times 10^{6}$ \\
\hline (i) 1744512 & 550 & 308 & 1.787 & 3.495 & 0.045 & $1.459 \times 10^{6}$ \\
\hline (j) 1622352 & 660 & 398 & 1.659 & 3.702 & 0.032 & $8.502 \times 10^{5}$ \\
\hline
\end{tabular}




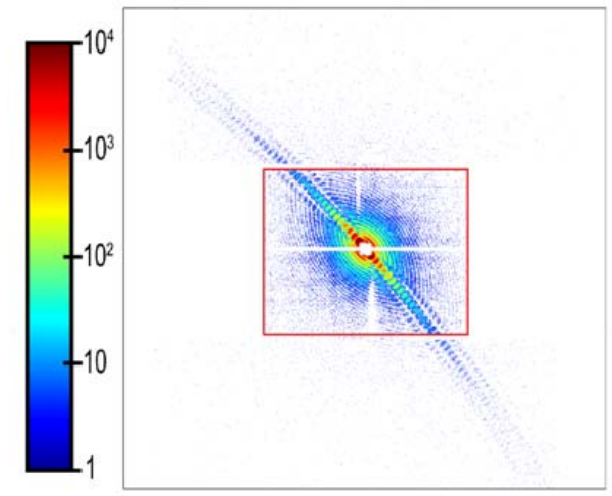

Fig. 5. Extended diffraction image derived by combining the rear detector data of Fig. 1 (h) (within red rectangle) with the appropriately scaled image from the front detector (outside the red rectangle). The logarithmic intensity color scale is shown on the left.

The diffraction image in Fig. 1 (h) is particularly intense and extends to sufficiently large scattering angles such that it is also detectable with the front CCD detector. It must stem from an event for which the imaged droplet was very close to the focus of the XFEL beam. Fig. 5 shows a composite image which was obtained by combining the data from the front and back detectors upon appropriate scaling. The data from the rear detector (same as that in Fig. 1 (h)) are contained within the red rectangle. The streak in Fig. 5 exhibits a noticeable curvature. In comparison, most of the other recorded streaks appear as straight stripes within the accuracy of

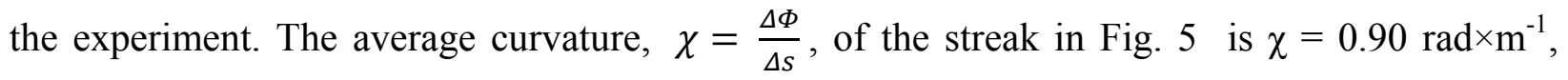
where $\Delta \Phi$ is the angular deviation of the streak from a straight line along its length $\Delta \mathrm{s}$. An additional distinction of the image in Fig. 5 is that it exhibits nodes (weak intensity region) between the streak and the diffraction rings, i.e. a discontinuity of the diffraction pattern, while most of the other observed streaks merge smoothly into the ring patterns at small scattering angles. Moreover, two satellite streaks emerge with increasing scattering angles in Fig. 5. 


\section{Shapes of classical rotating droplets}

For the purpose of assigning the observed diffraction images to particular droplet shapes it is instructive to review the deformations of rotating classical droplets. The equilibrium shape of a droplet rotating as rigid body is defined by the balance between capillary forces from surface tension and centrifugal forces. The stability diagram and corresponding representative shapes for axially symmetric $\left(D_{\infty \mathrm{h}}\right)$ and two-lobed $\left(\mathrm{D}_{2 h}\right)$ branches are shown in Fig. 6 [2, 4-7]. In Fig. 6 the reduced angular momentum, $\Lambda$, and reduced angular velocity, $\Omega$, are given by:

$$
\begin{aligned}
& \Lambda=\frac{1}{\sqrt{8 \cdot \sigma \cdot \rho \cdot R^{7}}} \cdot L, \\
& \Omega=\sqrt{\frac{\rho \cdot R^{3}}{8 \cdot \sigma}} \cdot \omega,
\end{aligned}
$$

where $L$ and $\omega$ are the angular momentum and angular velocity, respectively, in absolute units, $\sigma$ is the surface tension of the liquid, $\rho$ is the density of the liquid and $R$ is the radius of a spherical droplet with the same volume as the distorted droplet. Fig. 6 shows that, initially, upon increasing $\Lambda$, the equilibrium shape of the droplet evolves from spherical to oblate axially symmetric. At large $\Lambda$, the shapes show considerable flattening in the polar regions and even depressions at $\Lambda>2.03$. However, beyond $\Lambda \approx 1.2$ the axially symmetric shapes become unstable with respect to two-lobed deformations. At $\Lambda>1.2$ stable shapes are described by the lower branch representing prolate triaxial droplets, which resemble elongated pills at $1.2<\Lambda<1.6$, dumb-bell shapes at $\Lambda>1.6$ and eventually become unstable against fission at $\Lambda>2$. 


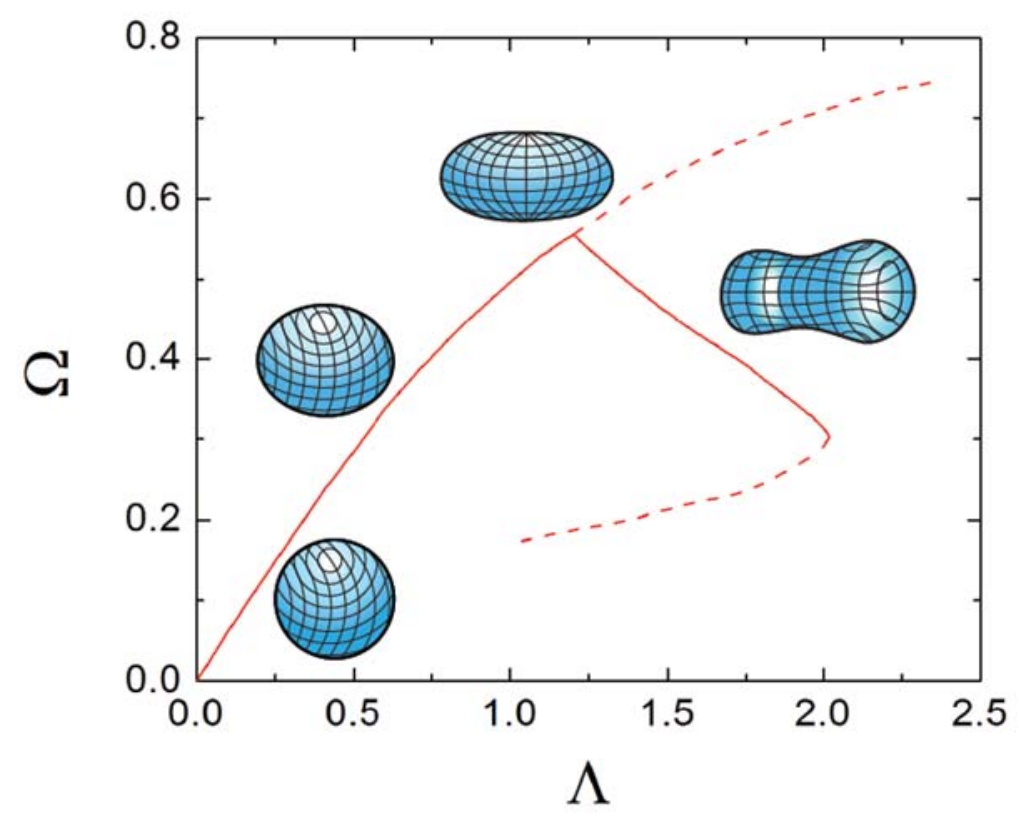

Fig. 6. Stability diagram for rotating droplets in equilibrium as a function of the reduced angular velocity, $\Omega$, and the reduced angular momentum, $\Lambda$, see eqs. $(1,2)$. The upper branch corresponds to oblate axisymmetric shapes, whereas the lower branch to prolate two-lobed shapes. The bifurcation point is located at $\Lambda=1.2, \Omega=0.56$ with $\mathrm{AR}=1.48$.

Cross sections containing the rotation axis of axially symmetric droplets at various values of $\Lambda$ are shown in Fig. 7 a), as calculated from equations in Ref. [4]. The droplets along the twolobed branch are triaxial bodies having $\mathrm{D}_{2 \mathrm{~h}}$ symmetry (a principal $\mathrm{C}_{2}$ axis, two $\mathrm{C}_{2}$ rotational axes perpendicular to the principal axis, a horizontal mirror plane perpendicular to the principal axis, and two vertical planes of symmetry). The equilibrium shapes of the prolate droplets at $1.2<\Lambda<$ 2 were calculated numerically [5-6]. The cross sections for the prolate shapes are shown in Fig. 7 b) and c). The rotation axis is aligned perpendicular to the figure plane in Fig. 7 b) and it is vertically aligned in the figure plane in Fig. 7 c). The longest semi-major axis ("long axis") of the triaxial shape is aligned horizontally in both Figs. 7 b) and c). The numerical data for the calculated oblate cross sections [4] and prolate [5-6] three dimensional surfaces are described in section S3 in SM and tabulated in the deposited EXCEL file. The droplets are characterized by 
the distance between the center of mass and the droplet's surface along the three mutually perpendicular directions; $a$ is the distance along the rotation axis, $c$ is the distance along the long axis, whereas $b$ is the intermediate distance. Corresponding aspect ratios, $\mathrm{AR},(c / a, b / a$ and $c / b)$ are shown in Fig. 8 a. The axially symmetric shape is characterized by a single AR of $b / a$. Fig. 8 b) illustrates the ratios of the droplet volume to the cube of the long half axis (given as multiples of $4 \pi / 3$ ) for axially symmetric oblate and two-lobed prolate shapes. The results in Fig. 8 a) and b) enable attaining the values of angular momentum, $L$, and angular velocity, $\omega$, of the droplets from the observed shapes.
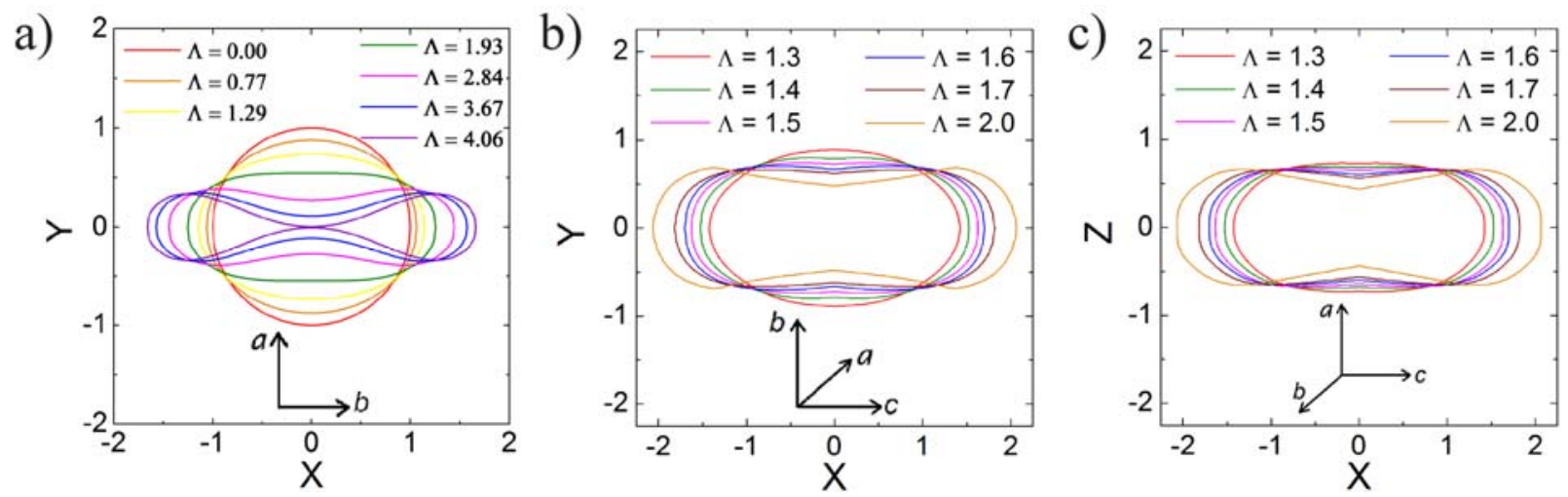

Fig. 7. Calculated cross sections of rotating classical droplets for various reduced angular momenta $\Lambda$ as indicated. The angular momentum is aligned along the short $a$-axis. The panels correspond to axisymmetric oblate shapes a), and two-lobed prolate shapes in the equatorial plane b) and in the plane containing the rotational axis and the long axis c). Note the different scales in a) and b, c). All droplets have the same volume of $4 \pi / 3$. 
a)

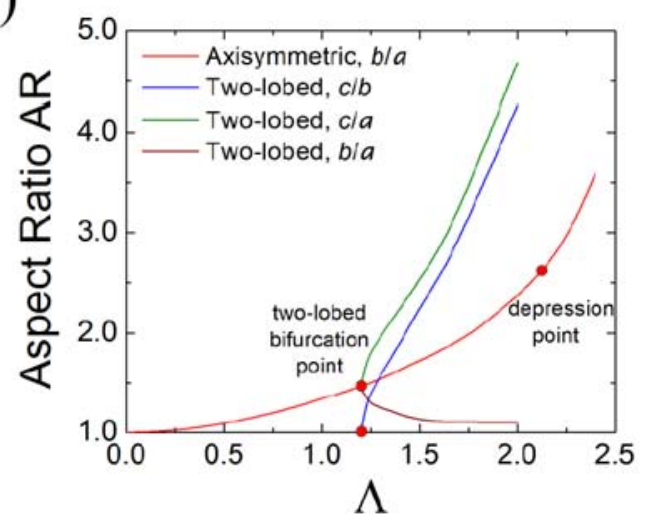

b)

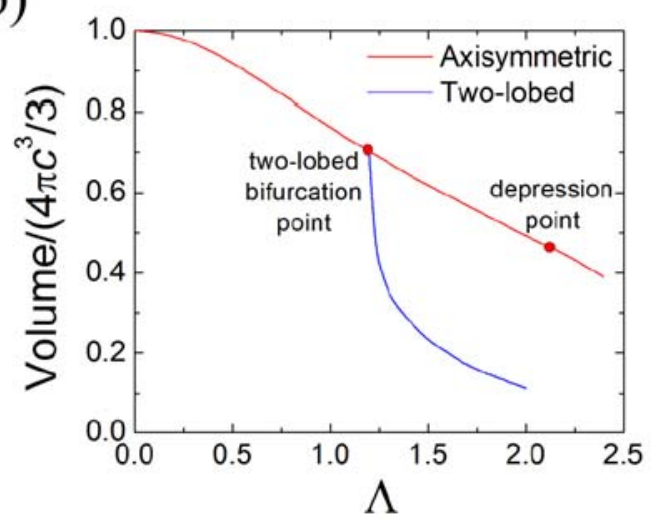

Fig. 8. a) Calculated aspect ratio, AR, vs. reduced angular momentum $\Lambda$ for classical axially symmetric oblate (red) and two-lobed prolate (green, blue, brown) droplet shapes. The color codes indicate the following aspect ratios: red: $b / a$; green: $c / a$; blue: $c / b$; brown: $b / a$. b) Ratio of the droplet volume to the cube of the c-axis for prolate shapes and b-axis for oblate shapes in units of $4 \pi / 3$. Red: axially symmetric oblate shapes; blue: two-lobed prolate shapes.

\section{Discussion}

The shape analysis for superfluid droplets presented here is based on small angle scattering data. The corresponding scattering theory is discussed in section S4 of the SM. It follows that at small angles the diffraction from a spheroid consists of elliptical rings with constant intensities along each specific ring. Whether a spheroidal droplet is oblate or prolate cannot be determined from small angle scattering experiments as the diffraction images yield only information on the projection of the droplet onto the detector plane. Accordingly, the reported image aspect ratios $(\mathrm{AR}>1)$ correspond to lower bounds of the actual droplet $\mathrm{AR}$ values.

In experimental runs 1 and 2, approximately 98\% of the obtained diffraction images from bare He droplets exhibit round or elliptical patterns with $\mathrm{AR}<1.3$ [18]. Those patterns were assigned to spherical or oblate spheroidal droplets. This assignment is in agreement with the range of stability of axisymmetric droplet shapes, whereas droplets having angular momenta 
beyond the bifurcation point of $\Lambda \approx 1.2$ have aspect ratios exceeding 1.48. Note that droplet shape oscillations are expected to decay long before the droplets reach the x-ray beam and are therefore unlikely candidates for the detected deviations from spherical droplet shapes. In the SM of Ref. [18] we estimated that the shape oscillations excited upon the creation of the droplets decay within a drift distance of about $1 \mathrm{~mm}$ from the nozzle due to viscous energy dissipation. At $\mathrm{T}<0.9 \mathrm{~K}$, the mean free path of phonons exceeds the radii of droplets relevant to this work. Under these conditions, the concept of viscosity is no longer applicable and energy dissipation is caused by interaction of ballistic phonons with surface ripplons. In the molecular regime, the lifetime of surface ripplons is given by: [34]

$$
\tau_{Q}=\frac{60 \cdot \rho}{\pi^{2} \cdot \hbar \cdot Q} \cdot\left(\frac{\hbar \cdot s}{k_{B} \cdot T}\right)^{4}
$$

where $\mathrm{Q}$ is the ripplon wavevector, and $\mathrm{s}$ the velocity of sound. In the droplet, $\mathrm{Q}$ can be expressed in terms of the oscillation mode multipole number $l:$ [35]

$$
Q=\frac{\sqrt[3]{l \cdot(l-1) \cdot(l+2)}}{R}
$$

Thus, the lifetime of droplet's shape oscillation is:

$$
\tau_{l}=\frac{60 \cdot \rho \cdot R}{\pi^{2} \cdot \hbar \cdot \sqrt[3]{l \cdot(l-1) \cdot(l+2)}} \cdot\left(\frac{\hbar \cdot s}{k_{B} \cdot T}\right)^{4}
$$

For example, for a droplet with $\mathrm{R}=300 \mathrm{~nm}$ and $\mathrm{T}=0.4 \mathrm{~K}$, the oscillation lifetime is $\tau_{2}=6 \times 10^{-4}$ $\mathrm{s}$, which is negligible compared to the time-of-flight of $4 \mathrm{~ms}$ to reach the interaction volume.

\subsection{X-ray scattering from strongly deformed He droplets}

The droplets at the focus of this work produce non-elliptic diffraction contours with pronounced intensity anomalies, most dramatically manifested in streaks, such as in Fig. 1 (f) - 
(j). The diffraction intensities $I \propto \theta^{-\alpha}$ decrease more slowly with increasing scattering angle $\theta$ along the streaks as compared to the rest of the diffraction images. Eqs. (S4.2 and S4.3) in section S4 in SM entails that, for a spheroid, the diffraction intensity scales with the scattering angle to the negative power of $\alpha=4$ (Porod's law), which is in agreement with the experimental observations. The same dependence is observed in the images with strongly deformed diffraction contours for azimuthal angles away from the long axis. However, for these images, the values of $\alpha$ are significantly smaller along the major axis of the diffraction image, as illustrated in Fig. 3. Along the streaks, the values of $\alpha$ vary between $\sim 3.2$ and $\sim 3.7$, signifying a considerable deviation of the droplet shapes from an ellipsoid. In general, a streak in the diffraction pattern emerges when rays originating from a group of points on the droplet surface possess the same path length difference. Examples for such groups of points are two opposite flat surfaces of a cylinder perpendicular to its axis or from two lines of points on the opposite sides of a cylinder parallel to its axis, where the values of $\alpha$ are 2 and 3, respectively.

The shapes of the droplets are related to their diffraction patterns via an inverse Fourier transformation, see eqs. (S4.2, S4.3) in SM section S4. For small scattering angles, $\theta$, the phase difference acquired due to the extension of the object along the $\mathrm{x}$-ray beam, $\mathrm{z}$, is described by:

$\Delta \varphi_{Z}=\frac{\pi \cdot z \cdot \theta^{2}}{\lambda}$

For a characteristic length of $\mathrm{z}=500 \mathrm{~nm}$ and a scattering angle $\theta<0.02 \mathrm{rad}$ (as in most of our images) $\Delta \varphi_{Z}<0.14 \cdot \pi$ which is small and the form factor in eq. (S4.3) in SM is well approximated by the two-dimensional Fourier transform of the projection of the density onto the detector plane. Therefore, we use an inverse two-dimensional Fourier transform of the diffraction 
amplitudes in order to obtain the corresponding droplet shapes, as described in detail in the section S5 in SM.

a)

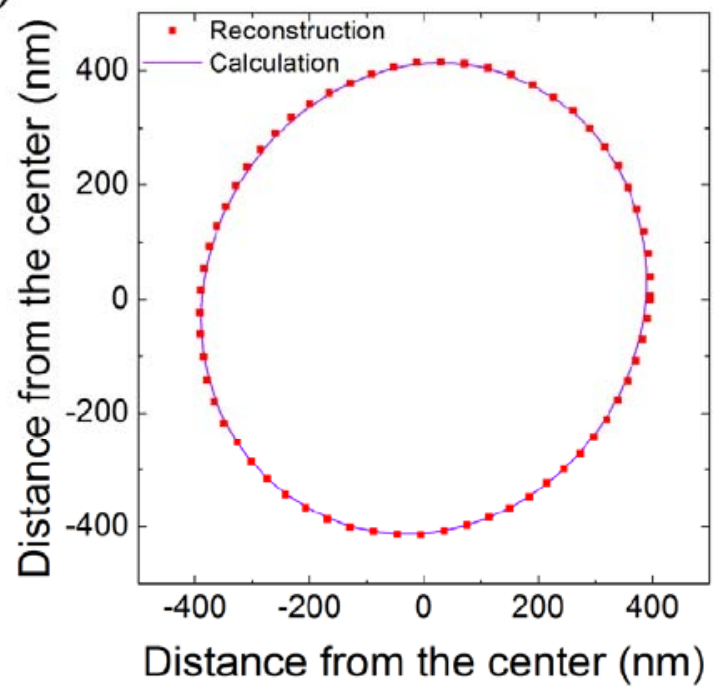

c)

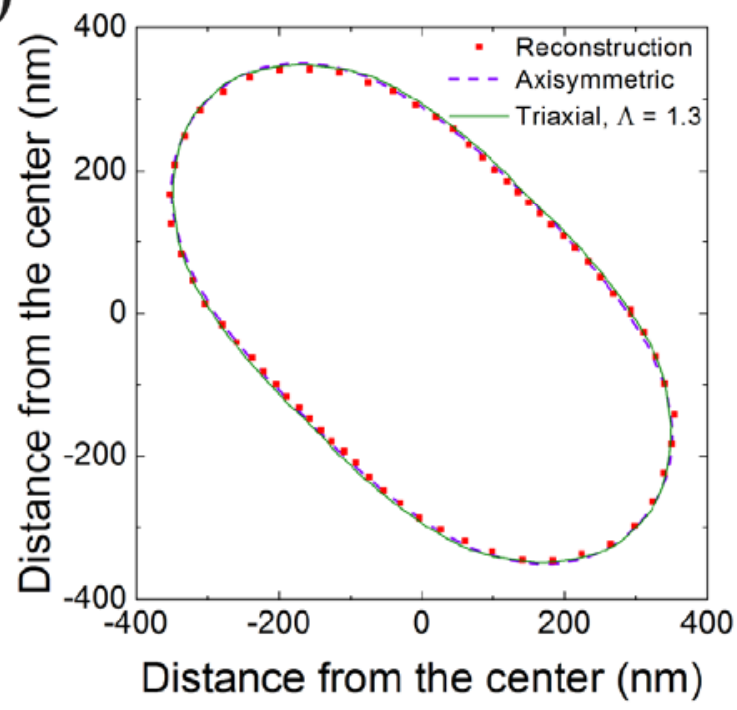

b)

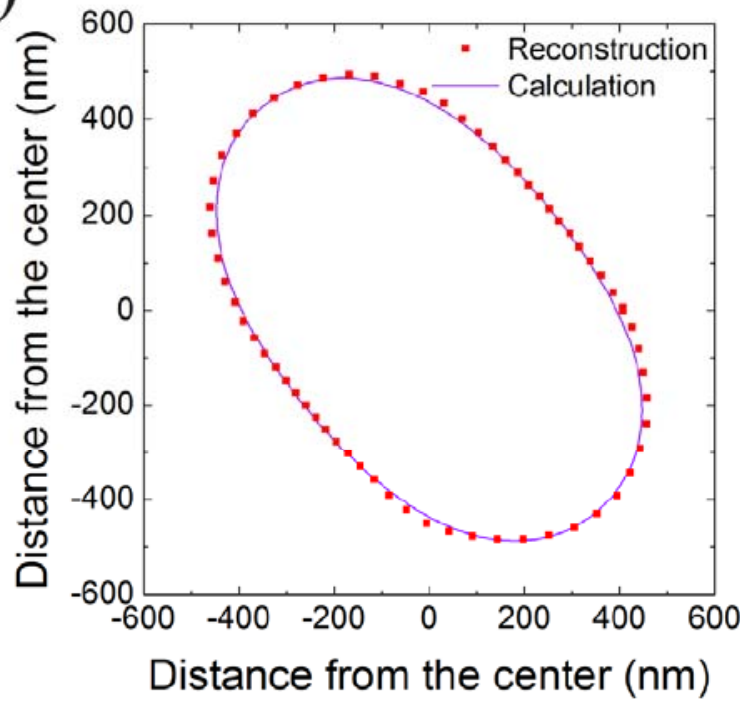

d)

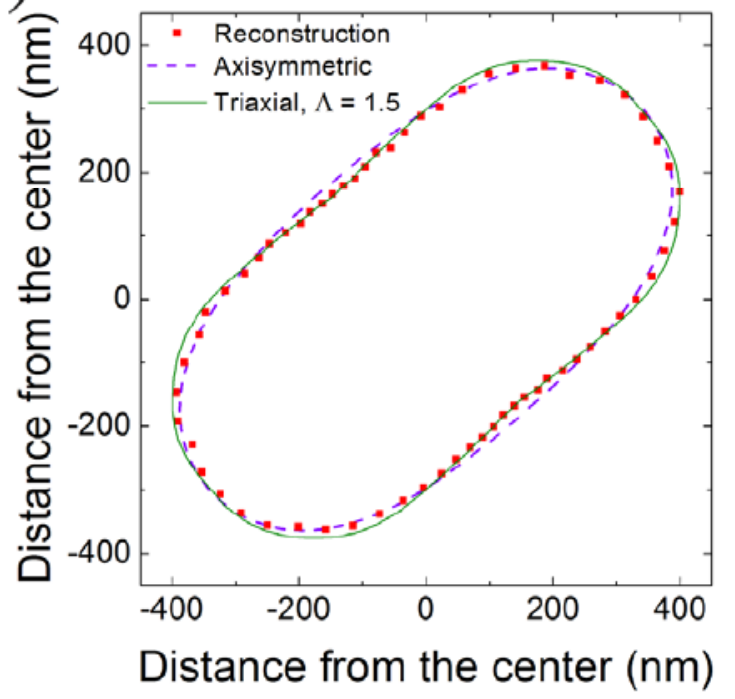

Fig. 9. Droplet contours obtained by inverse Fourier transformation of the diffraction images in Fig. 1 a), g) and h) are outlined by red squares in panels a), b) and d), respectively. Panel c) shows the inverse Fourier transform of the diffraction image in Fig. $2 \mathrm{C}$ of Ref. [18]. Violet curves are calculated contours for axially symmetric shapes with the same aspect ratio as found experimentally. The green contour in c) is the result of a calculation for a two-lobed shape with $\Lambda=1.3$ and a $9^{\circ}$ tilt of the rotation axis relative to the detector plane, whereas the one in d) is the result of a calculation for a two-lobed shape with $\Lambda=1.5$ and a $45^{\circ}$ tilt. 
The shapes obtained from the diffraction images in Fig. 1 a), g) and h) are outlined by red squares in Fig. 9 a), b) and d), respectively. Fig. 9 c) shows the shape obtained from the streaked diffraction image in Ref. [18]. Unfortunately, only the contours and not the entire density distribution of the droplets could be obtained from the diffraction data due to the lack of signals at very small scattering angles, which fall into the central hole of the detector. The droplet contour in Fig. 9 a) has an elliptic shape in agreement with the elliptic diffraction contours in Fig. 1 a). The contours in Fig. 9 b), c) are no longer elliptic and show pronounced regions of low curvature, where the opposite sides of the contours run nearly parallel. This behavior is consistent with the observed streaks as discussed earlier. Finally, the contour in Fig. 9 d) exhibits noticeable depressions within the parallel surfaces.

The violet curves in Fig. 9 a), b), c), and d) show the results of calculations [4] for axially symmetric oblate rotating droplets, such as those shown in Fig. 7 a), with the experimentally determined aspect ratios of $\mathrm{AR}=1.10,1.67,1.93$ and 1.92, respectively. The contours represent edge-on views of the droplets, i.e., with the axis of rotation in the figure plane. More diffraction images and reconstructed shapes are presented in section S1 in SM. Fig. 9 a) shows a good correspondence between the experimental and calculated contours, which have approximately elliptic shapes. The distorted experimental contours in Fig. 9 b), c) are also in good agreement with the calculations. Experimental and calculated contours in Fig. 9 d), however, exhibit considerable differences in that the calculated contour does not show any depression. The depression in the droplet manifests itself in the appearance of multiple diffraction streaks as discussed in relation to Fig. 5. Besides the recently recorded image in Fig. 5, only one additional image of the run 1 with $\mathrm{AR}=2.39$ had similar nodes (see image 10459657 in SM section S1), indicating a very small abundance of droplets with depressions in the beam. Previously, [18] we 
assigned contours such as in Fig. 9 c) to axially symmetric shapes imaged edge on based on the close resemblance to the expected shapes. However, axially symmetric shapes develop depressions only at very large $\Lambda>2.1$, and the aspect ratio for such droplets exceeds $\approx 2.5$, see Fig. 8 a). Accordingly, axially symmetric droplets are unlikely candidates for the contour observed in Fig. 9 d), which has an aspect ratio of 1.92.

We must therefore consider shapes along the two-lobed family in more detail. The presence of such shapes in the beam is consistent with the observation of the curved streak in Fig. 5. In the two-dimensional approximation, diffraction by a homogenous body of any shape gives rise to centro-symmetric diffraction images. The curved streak, therefore, signifies a departure from the two-dimensional approximation and indicates that the phase acquired along zdirection cannot be neglected (see eq. (6)) [36]. Diffraction images from tilted axially symmetric shapes obtained by three dimensional Fourier transforms are presented in section S6 in SM. It follows that diffraction with curved streaks could not be produced from an oblate axisymmetric shape at any tilt angle. On the other hand, curved diffraction streaks may naturally originate from prolate shapes that are tilted such that the x-ray beam is not contained in any of the object's planes of symmetry.

In order to gain more insight into the diffraction that can arise from two-lobed shapes, a large number of diffraction images have been calculated for each of the representative two-lobed shapes illustrated in Figs. 7 b), c). For this purpose the shapes were tilted around $a$ or $b$ axis and three dimensional Fourier transforms were calculated and presented in section S7 in SM. The calculations indicate that the triple streak as observed in Fig. 5 is consistent with droplets marked by small depressions and a reduced angular momentum of $\Lambda=1.50 \pm 0.05$. Shapes with $\Lambda<1.5$ have no depression and produce a single streak. Shapes with $\Lambda>1.5$ have a large depression and 
produce multiple streaks or even X-shaped streaks in the diffraction patterns, which were not observed in this work. The closest match with the experimental diffraction image is produced by a two-lobed droplet shape with $\Lambda=1.50$, with $c=550 \mathrm{~nm}$ and $a=230 \mathrm{~nm}$ tilted out of plane around the $a$ axis by $\pi / 4$. Fig. 10 shows a comparison of the measured and the simulated diffraction images, demonstrating very good agreement. The corresponding contour is shown by green curve in Fig. 9 d), which is in very good agreement with the experimental contour. Fig. 9 c) also shows the contour of the prolate droplet having the same AR with $\Lambda=1.30$, which is tilted around the $a$ axis by $9^{0}$. It is seen that the contours from axially symmetric oblate and prolate droplets both represent the experimental contour in Fig. 9 c) equally well. Therefore the contours alone could not be used to discriminate between the oblate and prolate droplets.

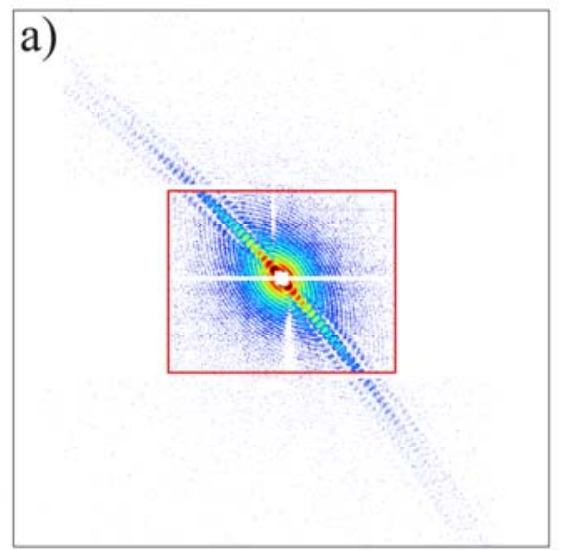

b)

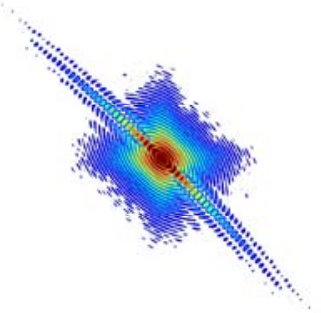

c)

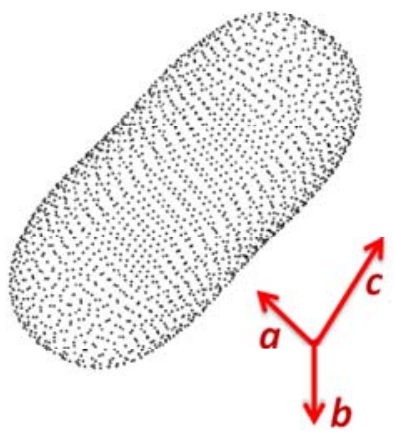

Fig. 10. Panel a) reproduces the image from Fig. 5. Panel b) shows a simulated diffraction image obtained from a two-lobe shape in panel c) with $\Lambda=1.5, c=550 \mathrm{~nm}$ and $a=230 \mathrm{~nm}$ tilted out of plane around the $a$ axis by $\pi / 4$. X-ray beam enters perpendicular to the plane of the figure. See the text for more details.

\subsection{Oblate and prolate shapes of rotating droplets}

The curved streaks in Figs. 5 and 10 a) provide direct evidence for the existence of prolate shapes in the droplet beam. However, at the wavelengths used in the LCLS experimental 
run 1 and $2(\lambda=1.46$ and $0.826 \mathrm{~nm})$ the vast majority of images, such as in Fig. 1, appear centrosymmetric. In particular, most of the streaks do not exhibit any curvature within the accuracy of the measurements. Note that this may reflect our inability to accurately determine streak curvatures at small scattering angles. The limited 3D shape information provided by the vast majority of the recorded small angle scattering data results in a significant uncertainty regarding the shapes of most droplets associated with streaked diffraction patterns. The results in sections S6 and S7 of the SM show that in addition to the axially symmetric oblate droplets, the diffraction images with single streaks (without side maxima, such as in Fig 5 and 10 a) ) could also originate from prolate shapes with $1.3<\Lambda<1.5$. Contours such as in Fig. 9 (c, d) would then be reproduced if one takes into account a possible tilt of the prolate shapes relative to the detector plane. Therefore, we need to reevaluate our previous assignment of the streaked diffraction images to axially symmetric oblate shapes [18]. Here, we analyze the appearance statistics of streaked images, which may contain information on the abundance of oblate and prolate shapes in the beam. The analysis is based on the fact that the appearance probability of streaks differs for oblate and prolate shapes at different tilt angles, which translates into different probabilities for the observation of streaks as a function of the shape aspect ratio. In the case of oblate axially symmetric shapes, streaks are only observed for very high aspect ratios and only if a droplet is imaged (nearly) edge-on with a tilt angle of no more than $\approx 10-15^{\circ}$. As a result, no streaks are expected for oblate droplets producing diffraction images with $\mathrm{AR}<1.6$. Vice versa, images with large AR devoid of any streaks will result from tilted axially symmetric oblate shapes. For prolate shapes, however, streaks may be observed at tilt angles as large as $80^{\circ}$, even in images with a small $\mathrm{AR} \approx 1.1$, as documented in section $\mathrm{S} 7$ in the $\mathrm{SM}$. 


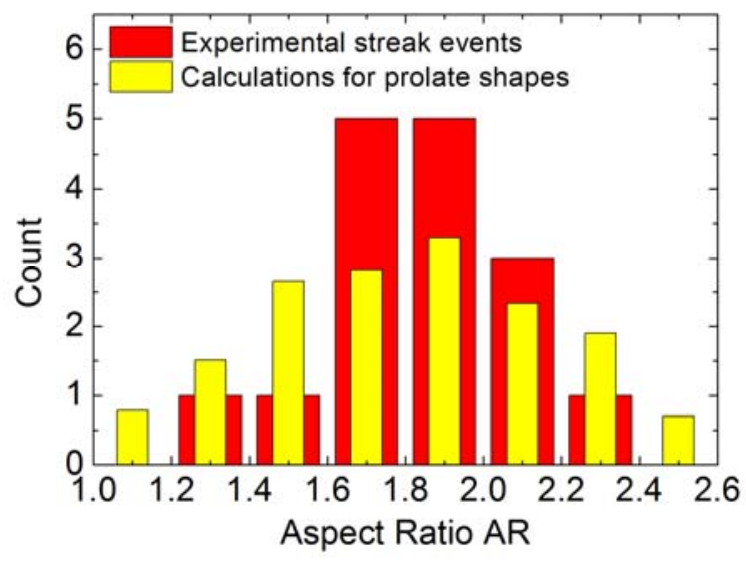

Fig. 11. Probability to observe streaked images with a certain aspect ratio for randomly orientated prolate droplets with $\Lambda=1.3,1.4$ or 1.5 (yellow). The three values of $\Lambda$ have been assigned the same probability. The experimentally observed streaked events (red) are the same as in Fig. 2, but sorted into larger bin sizes of 0.2.

Upon tilting a prolate droplet around the $a$ axis, its projection aspect ratio in the detector plane changes from its maximum value (when the droplet is viewed perpendicular to its long axis as in Fig. 7 c) to nearly 1 when the droplet is viewed along its long axis. Fig. 11 shows the calculated probability to observe a streaked image with an aspect ratio between 1.0 and 2.6 (binned into intervals of 0.2 ) for randomly oriented two-lobed droplets with $\Lambda=1.3,1.4$ or 1.5 (yellow). All three values of $\Lambda$ have been assigned the same probability. From Fig. 11 it is seen that there is a considerable probability of $\sim 31 \%$ to find a diffraction pattern with a streak and a small $\mathrm{AR}<1.6$ (based on the estimate that the vast majority of diffraction patterns from randomly oriented prolate droplets contain streaks, see section S7 in SM). This prediction may be compared with the experimental results in Fig. 11 (red). If all of the streaked images would stem from prolate droplets, the total of 14 streaked images with $\mathrm{AR}>1.6$ should be complemented by about 6 streaks with AR $<1.6$, whereas only 2 are observed. Although the number of counts is relatively modest, this discrepancy indicates that prolate shapes alone cannot 
account for all of the experimental deficiency of small AR events in streaked images. Consequently, in addition to prolate shapes, the distribution may contain axisymmetric oblate shapes, as we have previously postulated [18].

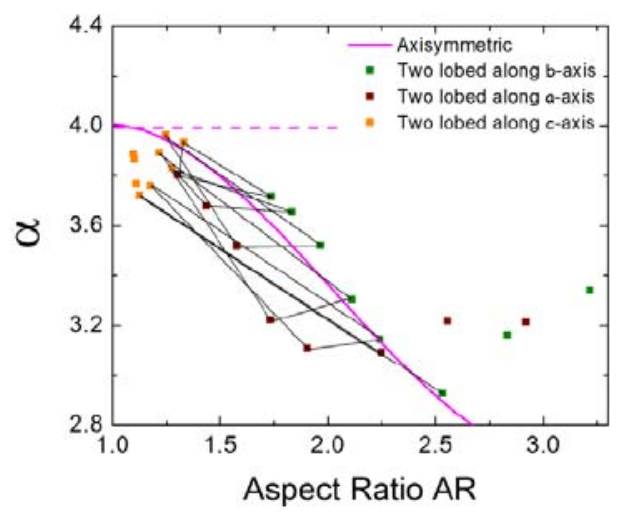

Fig. 12. Calculated values of decay exponent $\alpha$ versus AR for axially symmetric droplets imaged edge on (pink curve) and for two-lobed shapes imaged along their semi-axes $a, b$, and $c$ (see Fig. $7 \mathrm{~b}$ ), c) for axis orientations). The calculated two-lobed shapes have $\Lambda=1.23,1.26 .1 .3,1.35,1.4$, 1.5, 1.6, 1.7 in order of increasing AR. Points corresponding to the same shapes are connected with lines, i.e., each triangle corresponds to one shape imaged along three different axes.

Another important piece of information on the abundance of different shapes is provided by analyzing the power dependence of the diffraction intensity along the streaks as quantified by the decay exponents, $\alpha$, in Fig. 3. Fig. 12 summarizes the calculated values of $\alpha$ for prolate and oblate shapes with various ARs and orientations with respect to the x-ray beam. Square symbols show $(\mathrm{AR}, \alpha)$ pairs calculated for shapes with $\Lambda=1.23,1.26,1.3,1.35,1.4,1.5,1.6,1.7$ and 2.0 as shown in Fig. 7 b), c). For each shape, three differently colored squares indicate three different orientations of the shape with respect to the x-ray beam: orange - long axis, $c$, parallel to the $\mathrm{x}$-ray beam; green - intermediate axis, $b$, parallel to the $\mathrm{x}$-ray beam; brown -short (rotation) axis, $a$, parallel to the x-ray beam. The lines in Fig. 12 connect data points for shapes 
with the same value of $\Lambda=1.23,1.26,1.3,1.35,1.4,1.5$. The resulting triangles approximately delimit the locus of $(\mathrm{AR}, \alpha)$ points that may be obtained from a given shape with a particular $\Lambda$ and at an arbitrary orientation with respect to the X-ray beam. Data points for $\Lambda=1.6,1.7$, and 2.0 are shown for additional reference but not connected by lines, since no indication for corresponding droplet shapes were detected experimentally (see below). The continuous pink curves in Figs. 3 and 12 indicate the computed (AR, $\alpha)$ relationship for classical axisymmetric shapes placed with their short axis perpendicular to the X-ray beam (edge on) with $\Lambda$ varying from 0 to 2 . As discussed earlier, upon a $10-15^{\circ}$ tilt of the axially symmetric oblate shapes, the streaks in the diffraction patterns disappear, the values of $\alpha$ approach 4 (as indicated by the horizontal pink dashed line in Figs. 3 and 12) and the AR decreases. Therefore the (AR, $\alpha)$ points for axially symmetric shapes at arbitrary orientations fill the space between the pink continuous curve and the pink dashed line at $\alpha=4$. In contrast, Fig. 12 shows that the (AR, $\alpha$ ) points for prolate shapes reside mainly below the pink continuous curve. These differences can be utilized to classify the observed diffraction events into axially symmetric oblate or two-lobed prolate droplets.

A closer inspection of Fig. 3 shows that a number of (AR, $\alpha)$ values for streaked events are well below the axisymmetric curve and are consistent with values expected for two-lobed shapes having $\Lambda$ in the range 1.26 - 1.5. At larger $\Lambda=1.6$ and 1.7 (unconnected squares in Fig. 12), the droplets have some pronounced depressions, leading to multiple streaks in the diffraction patterns, see section S7 in the SM. Such diffraction images, however, were not recorded experimentally, suggesting that the probability for formation of droplets with $\Lambda>1.5$ in the presented experiments is very low. On the other hand, Fig. 3 indicates a large number of events with AR in the range of $1.5-1.85$ and $\alpha \approx 4$ within an uncertainty of about \pm 0.2 . These points are 
located significantly higher in the $(\mathrm{AR}, \alpha)$ correlation plot than the range expected for prolate shapes. The ARs of these events are beyond those of stable, axially symmetric shapes with $\Lambda=$ 1.2, $\mathrm{AR}=1.48$ (see Fig. 6). Therefore, these points indicate the existence of axially symmetric shapes with $\Lambda=1.2-1.5$, which are beyond the stability range of classical rotating droplets as discussed previously [18]. Finally, the origin of the points in Fig. 3 with AR $>1.9$ remains uncertain. These points are located far away from the range expected to contain two-lobed shapes. However, the absence of large aspect ratio events in Fig. 3 with AR $>1.9$ makes axially symmetric shapes unlikely candidates for these points.

As discussed in Section 5.1, the full three-dimensional shapes of the imaged droplets could not be reconstructed from the small angle scattering data presented in this work. Therefore, oblate droplets may also deviate from axial symmetry, the extent of these deviations, however, cannot be quantified. More accurate information on the droplet shapes could be obtained from measurements at large scattering angles, where the deviation of the diffraction images from centro-symmetric patterns may be used to quantify the droplet's shape. This, however, would require using a much longer wavelength of radiation of about $12 \mathrm{~nm}(100 \mathrm{eV})$ to assure sufficiently strong scattering at large angles. This long wavelength regime is not available at LCLS, but is common at the FLASH and FERMI free electron lasers [37-38]. A recent XFEL based wide angle x-ray scattering study of Ag nano-particles enabled the reconstruction of their three-dimensional shapes [36]. Very recently, wide angle scattering from He droplets in the XUV regime has been studied [39].

The good agreement between the experimental and calculated droplet contours in Fig. 9 and diffraction patterns in Fig. 10 indicates that the classical calculations give a reasonable representation of the shapes of superfluid helium droplets. From the shapes, the angular velocity, 
$\omega$, and the angular momentum, $\mathrm{L}$, of the droplets can be estimated using eqs. $(1,2)$ and Figs. $6,8$. For example, the diffraction pattern in Fig. 10 likely stems from a prolate shape with $\Lambda \approx 1.5$ and a semi-major axis $a \approx 550 \mathrm{~nm}$ as discussed earlier. The resulting values of $\omega, \mathrm{L}$, as well as other parameters of the droplet are presented in the second column of the Table 2. For comparison, the same parameters have been calculated for axially symmetric droplets of the same volume rotating with $\Lambda=1.5$ (unstable) and $\Lambda=1.2$ (boundary stable/unstable). Table 2 also lists the values of $\mathrm{L}$ in units of $\mathrm{h}$ per droplet as well as per He atom in the droplet. These estimates show that the rotating droplet is marked by a very large angular momentum of $\approx 58 \hbar$ per single $\mathrm{He}$ atom and thus must contain a large number of quantum vortices. Obtaining the precise number of vortices, $N_{V}$, in a droplet of a given shape and angular momentum requires sophisticated calculations that are beyond the current state-of-the-art [21, 40-42]. Therefore, we estimate $N_{V}$ based on a two-dimensional model. Within this model, the number density of vortices is $n_{V}=\frac{2 \cdot \omega}{\kappa}$ and the number of vortices can be estimated as $N_{V}=n_{V} \cdot A$, where $A$ is the area of the equatorial cross section of the droplet, see Table 2 . This expression likely underestimates $N_{V}$ because vortices near the equator are shorter than those in the interior, and thus carry smaller angular momentum $[13,15]$. 
Table 2. Parameters of a prolate shape with $\Lambda=1.5$ and axisymmetric oblate shapes with $\Lambda=1.2$ and $\Lambda=1.5$ having the same volume of $1.63 \times 10^{8} \mathrm{~nm}^{3}\left(\mathrm{~N}_{\mathrm{He}}=3.56 \times 10^{9}, R_{\text {spherical }}=339 \mathrm{~nm}\right)$.

\begin{tabular}{|l|l|l|l|}
\hline & $\Lambda=1.5$ (prolate) & $\Lambda=1.2$ (oblate) & $\Lambda=1.5$ (oblate) \\
\hline Half major axis $(\mathrm{nm})$ & 550 & 372 & 387 \\
\hline Moment of inertia $\left(\mathrm{kg} . \mathrm{m}^{2}\right)$ & $4.061 \times 10^{-30}$ & $1.846 \times 10^{-30}$ & $2.050 \times 10^{-30}$ \\
\hline $\mathrm{L}(\hbar)$ & $2.064 \times 10^{11}$ & $1.651 \times 10^{11}$ & $2.064 \times 10^{11}$ \\
\hline $\mathrm{L}(\hbar) / \mathrm{N}_{\mathrm{He}}$ & 58 & 46 & 58 \\
\hline$\omega(\mathrm{rad} / \mathrm{s})$ & $1.028 \times 10^{7}$ & $1.245 \times 10^{7}$ & $1.409 \times 10^{7}$ \\
\hline Number of vortices & 122 & 108 & 133 \\
\hline Equatorial area $\left(\mathrm{nm}^{2}\right)$ & $5.939 \times 10^{5}$ & $4.347 \times 10^{5}$ & $4.705 \times 10^{5}$ \\
\hline
\end{tabular}

In general, in the presence of a large number of vortices, the equilibrium shapes of rotating superfluid droplets are expected to resemble classical droplets rotating at the same $\omega$ due to the similarities of the velocity fields far away from the vortex cores. For example, the shape of a rotating superfluid in a cylindrical container adopts a parabolic shape similar to that of a classically rotating liquid [10-11]. However, the question remains how accurately the classical shapes and the obtained classical $\omega$ describe rotating superfluid droplets. The relation between kinetic energy and angular momentum is different for classical droplets executing RBR and superfluid droplets containing a collection of vortices, which will most likely result in some differences of the droplets' shapes. For example, a droplet with $\mathrm{R}=340 \mathrm{~nm}$ containing a single central rectilinear vortex will have an $\mathrm{AR}=1.0022$ [15]. For comparison, a classical droplet with same angular momentum will have a smaller $\mathrm{AR}=1.00024$. This effect can be rationalized in terms of the filament tension; in order to minimize the lengths of the filaments, superfluid droplets adopt somewhat flatter shapes in the polar regions. Evidently, the distortions of droplets relevant to this work and containing a single vortex are negligible and currently below the detection limit of the method $(\sim 1 \%)$. The deviations between classical and superfluid droplet 
shapes for droplets containing multiple vortices, cannot be estimated in a straightforward manner, because the shape and the configuration of vortices are interrelated. It would be desirable to obtain the shapes of superfluid droplets and the corresponding vortex configurations as a function of $\mathrm{L}$ and $\mathrm{N}_{\mathrm{He}}$ in order to assemble the stability diagram for superfluid droplets, as has been established for their classical counterparts (see Fig. 6). Our measurements [18] indicate that in axially symmetric pseudo-spheroidal droplets vortices form an equilateral triangular lattice similar to those observed in Bose-Einstein condensates [12, 43]. In the future, similar measurements may help evaluating the arrangement of vortices in strongly deformed helium droplets.

\subsection{Formation of rotating droplets in the free jet}

In this Section, we will discuss possible origins of the angular momentum and shape deformations in helium droplets. The droplets are produced upon expansion of liquid helium at $\mathrm{T}_{0}=5 \mathrm{~K}$ and $\mathrm{P}_{0}=20$ bar into vacuum through a nozzle with a nominal diameter of $\mathrm{d}=5 \mu \mathrm{m}$ and a channel length of $2 \mu \mathrm{m}$. During the expansion of the fluid through the nozzle its temperature and pressure drop and the liquid is accelerated from rest to about $170 \mathrm{~m} / \mathrm{s}$ as experimentally determined [29]. If the fluid phase persists while reaching the vacuum, a jet is formed that boils vigorously giving rise to the formation of droplets. Estimates based on the rate of evaporative cooling show that a droplet with a $500 \mathrm{~nm}$ radius passes the superfluid phase transition within less than $\approx 1 \mu$ s and continues to cool to $\sim 0.4 \mathrm{~K}$ [44-45] well before reaching the X-ray focal point after about $4 \mathrm{~ms}$ time-of-flight. During the passage of the fluid helium through the nozzle it interacts with the channel walls and acquires vorticity, which is eventually transferred to the droplets. The precise form of the vorticity field in the nozzle is unknown. For example, it is conceivable that classical vortices are formed during the expansion. The magnitude of the 
vorticity may also depend on the unknown microscopic structure of the nozzle channel, which is manufactured (Plano) by mechanical drilling and may have some roughness on a sub-micron scale. While different nozzles were used during experimental runs 1 and 2, similar abundances for large AR and streaked events have been observed, indicating that the microscopic details of the nozzle channel may not play a dominant role. Thus, the order of a magnitude of the droplets' angular velocity may be estimated based on the nozzle diameter of $5 \mu \mathrm{m}$ and the measured terminal droplet velocity. Assuming that the fluid at the center of the nozzle moves with $v=170$ $\mathrm{m} / \mathrm{s}$ whereas it is at rest at the walls, the average velocity gradient gives an upper boundary for the droplet's angular velocity of about $\omega=\mathrm{v} / \mathrm{d} \approx 3 \times 10^{7} \mathrm{~s}^{-1}$. The angular momentum of a spherical droplet of radius $\mathrm{R}$ is $\mathrm{L}=(8 / 15) \times \pi \times \rho \times \mathrm{R}^{5} \times \omega=9 \times 10^{-2} \times \mathrm{R}(\mathrm{nm})^{5}$ ( $)$. This value can be compared with the critical angular momentum for disintegration of the droplets [6] $L_{\mathrm{CRIT}} \approx$ $2 \times\left(8 \times \sigma \times \rho \times R^{7}\right)^{1 / 2}=410 \times R(n m)^{7 / 2}$ (ћ). It follows that droplets with $\mathrm{R}>300 \mathrm{~nm}$ cannot sustain the maximum vorticity and will undergo fission. Here, we have used the density $\rho=145 \mathrm{~kg} / \mathrm{m}^{3}$ [46] and surface tension $\sigma=3.54 \times 10^{-4} \mathrm{~N} / \mathrm{m}[46]$ of liquid helium at low temperatures. The relevant temperature in the nozzle expansion is likely about $4 \mathrm{~K}$ with a smaller $\sigma=1.1 \times 10^{-4} \mathrm{~N} / \mathrm{m}$ [46], which will further reduce the estimated value of $R$. As discussed previously [29], at $T_{0} \approx 5 \mathrm{~K}$ the fluid may separate into large droplets and a dense gas inside the nozzle, which continues to expand along the nozzle channel. Thus, if the droplets are produced before exiting from the nozzle, collisions with walls may contribute to the calculus of the droplet's angular momentum. It follows that the observed vorticity in a droplet is the result of a complex process that may include fission and collisions of the droplets.

The spontaneous formation of vortices during the rapid superfluid transition in bulk liquid He has attracted considerable attention as a model of the creation of cosmic strings during 
the early expansion of the Universe. [47] This so-called Kibble-Zurek mechanism was later supported by experiments in superfluid ${ }^{3} \mathrm{He}$, see [48-49] and references therein. In this work, however, in view of the preceding discussion the vorticity is more likely acquired during the flow of normal fluid helium through the nozzle channel.

In vacuum the droplets experience extensive evaporation. For example, cooling of a droplet from $4 \mathrm{~K}$ to $0.4 \mathrm{~K}$ results in the evaporation of about $30 \%$ of its atoms. The effect of the evaporation on the droplet's angular momentum remains to be studied. In a classical droplet the surface has higher angular momentum per atom, thus evaporation will likely lead to the reduction of the angular momentum per atom upon cooling. Nevertheless, the estimated maximum angular velocity of about $\approx 3 \times 10^{7} \mathrm{~s}^{-1}$ has the same order of magnitude and is slightly larger than the values obtained from the shapes of strongly distorted droplets of $\approx 10^{7} \mathrm{~s}^{-1}$, see Table 2. We also note that about $90 \%$ of the droplets in the beam have AR $<1.1$ and must predominantly originate from axially symmetric oblate droplets with $\Lambda<0.2$.

\subsection{Quadrupolar deformations and shapes of the rotating droplets}

The analysis of the strongly deformed diffraction images in Section 5.2 indicates the presence of the oblate droplets, which were identified with metastable axially symmetric shapes beyond the bifurcation point. Such shapes correspond to the dashed section of the upper branch in the stability diagram in Fig. 6. At $\mathrm{AR}>1.48$ the stable solutions correspond to two-lobed prolate shapes along the lower branch of the diagram in Fig. 6, which have lower rotational energy $\left(E_{\mathrm{ROT}}=\omega \times \mathrm{L} / 2\right)$. The transition from the upper to the lower branch requires energy dissipation, which in classical droplets is facilitated by viscosity. On the other hand, in superfluid droplets at low temperatures energy transfer due to viscosity may be negligible and axially 
symmetric shapes may persist longer than the time-of-flight of the droplets of about $4 \mathrm{~ms}$ as predicted previously [4].

The characteristic time for developing prolate shapes in rotating classical droplets may be estimated from the results of recent numerical Navier-Stokes calculations [6]. In these calculations, a spherical droplet of radius $\mathrm{R}$ was assumed to have a specific angular momentum and the time evolution of its shape was calculated while keeping the angular momentum constant. The calculations are valid for droplets with an Ohnesorge number $O_{h}=\eta \sqrt{\frac{\rho}{2 \cdot \sigma \cdot R}} \geq 1$, which corresponds to the range of overdamped oscillations [6]. Here $\eta$ is the kinematic viscosity of the liquid. The calculations indicated that a rotating droplet at $\Lambda>1.2$ first develops an axially symmetric oblate deformation, which remains metastable for a time $\tau_{1}$. At later times, the droplet becomes noticeably triaxial and eventually reaches a stationary prolate two-lobed shape at $\tau_{2}$. Using $O_{h}=1, \mathrm{R}=300 \mathrm{~nm}, \rho=145 \mathrm{~kg} / \mathrm{m}^{3}$, and $\sigma=3.5 \times 10^{-4} \mathrm{~N} / \mathrm{m}$, characteristic time scales of $\tau_{1}$ $\approx 7 \times 10^{-7} \mathrm{~s}$ and $\tau_{2} \approx 3 \times 10^{-6} \mathrm{~s}$ are obtained, which are of the same order of magnitude as the quadrupole oscillation damping time $\tau_{Q}=\frac{R^{2}}{5 \cdot \eta}=2 \times 10^{-6} \mathrm{~s}$. In comparison, the period for an underdamped quadrupole oscillation in $\mathrm{R}=300 \mathrm{~nm}$ droplets is $2.3 \times 10^{-7} \mathrm{~s}$. Unfortunately, similar calculations could not yet be performed for $O_{h}=0.008$ as in helium droplets, which follows using the kinematic viscosity of liquid helium at $\mathrm{T}=1.3 \mathrm{~K}$ of about $\eta=10^{-8} \mathrm{~m}^{2} / \mathrm{s}$ [46]. Similar magnitudes of $\tau_{1,2}$ and $\tau_{\mathrm{Q}}$ are not surprising because the deformation of an axially symmetric droplet involves coupling of the rotational motion with the quadrupolar deformation mode [4]. One can speculate that even at $O_{h}<<1$ the timescale for developing a prolate shape will be of the same order of magnitude as $\tau_{\mathrm{Q}}$, which is much shorter than the time of flight of the droplets from 
the nozzle to the x-ray interaction point. Thus, classical calculations are unlikely to explain the observation of oblate droplets with $\mathrm{AR}>1.5$ in this work.

The theory of quenching/development of shape deformations in this regime remains to be developed. In addition, quantum vortices in rotating superfluid droplets may lend additional stability to axially symmetric shapes as indicated by recent DFT calculations [21]. Therefore, the classical Navier Stokes equations may have limited applicability for the description of deformation kinetics in superfluid droplets.

\section{Conclusions}

This work reports the first systematic study of centrifugal deformations in submicrometer sized superfluid ${ }^{4} \mathrm{He}$ droplets by $\mathrm{x}$-ray coherent diffractive imaging. Strongly deformed droplets with aspect ratios up to 2.4 have been identified. The analysis of the images shows that in addition to axially symmetric oblate shapes, some droplets have triaxial prolate shapes. The obtained images can be well modeled by simulated x-ray diffraction patterns of classical droplet equilibrium shapes that belong to the axially symmetric and the two-lobed shape families. It follows that the shapes of rotating sub-micron superfluid droplets are very similar to their classical counterparts, which enables quantitative estimates for the angular momenta and angular velocities of the studied droplets. The results confirm the existence of oblate metastable superfluid droplets with large angular momenta beyond the stability range of classical droplets.

Since the measurements in this work were performed at small scattering angles, the full three dimensional droplet shapes could not be attained. Instead, the diffraction patterns only allow the reconstruction of the contours of the projection of the droplets onto the detector plane. 
On the other hand, classical calculations indicate that oblate axially symmetric droplets and nonaxially symmetric prolate droplets may have very similar contours. Moreover, the degree of possible deviations from axial symmetry in the oblate droplets cannot be quantified. As a result, the distinction between oblate and prolate droplets is based on differences in the power law that describes the scattering-angle dependent diffraction intensity as well as on statistical arguments. More accurate information on the droplet shapes may be obtained based on measurements at large scattering angles, where the deviation of diffraction images from centro-symmetric patterns may be used to quantify the droplet shapes. This, however, would require using a much longer wavelength of radiation of about $12 \mathrm{~nm}(100 \mathrm{eV})$, which has not been available for the presented experiments.

The interpretation of the results in this work is based on calculations of the equilibrium shapes of classical droplets rotating as rigid bodies. In superfluid droplets the liquid is not stationary in the rotating frame because its motion is determined by a collection of quantum vortices. The presence of quantum vortices should cause a deviation of the droplet shapes from their classical counterparts, which remains to be quantified. In the future it would be desirable to obtain the shapes of superfluid droplets and the configurations of vortices as a function of angular momentum and droplet size in order to assemble a stability diagram for superfluid droplets, as has been established for their classical counterparts. Our measurements [18] indicate that in axially symmetric oblate droplets, vortices form equilateral triangular lattices similar to those observed in Bose-Einstein condensates [12, 43]. Similar measurements may help evaluating the arrangements of vortices in strongly deformed prolate helium droplets. 


\section{Acknowledgements}

This work was supported by the NSF Grants No DMR-1501276 and CHE-1362535 (A.F.V.), by

the U.S. Department of Energy, Office of Basic Energy Sciences, (DOE, OBES) Chemical

Sciences, Geosciences and Biosciences Division, through Contract No. DE-AC02-05CH11231

(C.Ba., M.P.Z., A.S.C., O.G.) as well as DE-AC02-06CH11357 (M.B., C.Bo.) and DE-AC02-

76SF00515 (C.Bo.). Portions of this research were carried out at the Linac Coherent Light

Source, a national user facility operated by Stanford University on behalf of the U.S. DOE,

OBES under beam-time grant LA1214: Time-resolved imaging of X-ray induced dynamics in pure and embedded Xenon clusters.

\section{References}

1. S. Chandrasekhar, Ellipsoidal Figures of Equilibrium - an Historical Account, Commun. Pur. Appl. Math. 20, 251 (1967).

2. S. Cohen, F. Plasil, and W. J. Swiatecki, Equilibrium Configurations of Rotating Charged or Gravitating Liquid Masses with Surface-Tension. 2., Annals of Physics 82, 557 (1974).

3. N. Bohr, and J. A. Wheeler, The mechanism of fission, Phys. Rev. 56, 426 (1939).

4. S. Chandrasekhar, The stability of a rotating liquid drop, Proc. Roy. Soc. London A 286, 1 (1965).

5. R. A. Brown, and L. E. Scriven, The Shape and Stability of Rotating Liquid-Drops, Proc. R. Soc. A 371, 331 (1980).

6. S. L. Butler, M. R. Stauffer, G. Sinha, A. Lilly, and R. J. Spiteri, The shape distribution of splashform tektites predicted by numerical simulations of rotating fluid drops, J Fluid Mech 667, 358 (2011).

7. K. A. Baldwin, S. L. Butler, and R. J. A. Hill, Artificial tektites: an experimental technique for capturing the shapes of spinning drops, Sci Rep-Uk 5, 7660 (2015).

8. R. J. A. Hill, and L. Eaves, Nonaxisymmetric Shapes of a Magnetically Levitated and Spinning Water Droplet, Phys. Rev. Lett. 101, 234501 (2008).

9. R. P. Feynman, Application of quantum mechanics to liquid helium, in Progress in Low Temperature Physics, edited by C. J. Gorter (North-Holland Publishing Company, Amsterdam, 1955), Vol. 1, pp. 1.

10. R. J. Donnelly, Quantized Vortices in Helium II, Cambridge University Press, Cambridge, 1991).

11. D. R. Tilley, and J. Tilley, Superfluidity and Superconductivity, Institute of Physics Publ., Bristol, 1990).

12. L. Pitaevskii, and S. Stringari, Bose-Einstein Condensation, Clarendon Press, Oxford, 2003). 
13. G. H. Bauer, R. J. Donnelly, and W. F. Vinen, Vortex Configurations in a Freely Rotating Superfluid Drop, J. Low Temp. Phys. 98, 47 (1995).

14. S. T. Nam, G. H. Bauer, and R. J. Donnelly, Vortex patterns in a freely rotating superfluid, J Korean Phys Soc 29, 755 (1996).

15. K. K. Lehmann, and R. Schmied, Energetics and possible formation and decay mechanisms of vortices in helium nanodroplets, Phys. Rev. B 68, 224520 (2003).

16. M. A. Weilert, D. L. Whitaker, H. J. Maris, and G. M. Seidel, Magnetic levitation and noncoalescence of liquid helium, Phys. Rev. Lett. 77, 4840 (1996).

17. D. L. Whitaker, M. A. Weilert, C. L. Vicente, H. J. Maris, and G. M. Seidel, Oscillations of charged helium II drops, J. Low Temp. Phys. 110, 173 (1998).

18. L. F. Gomez, K. R. Ferguson, J. P. Cryan, C. Bacellar, R. M. P. Tanyag, C. Jones, S. Schorb, D. Anielski, A. Belkacem, C. Bernando, R. Boll, J. Bozek, S. Carron, G. Chen, T. Delmas, L. Englert, S. W. Epp, B. Erk, L. Foucar, R. Hartmann, A. Hexemer, M. Huth, J. Kwok, S. R. Leone, J. H. S. Ma, F. R. N. C. Maia, E. Malmerberg, S. Marchesini, D. M. Neumark, B. Poon, J. Prell, D. Rolles, B. Rudek, A. Rudenko, M. Seifrid, K. R. Siefermann, F. P. Sturm, M. Swiggers, J. Ullrich, F. Weise, P. Zwart, C. Bostedt, O. Gessner, and A. F. Vilesov, Shapes and vorticities of superfluid helium nanodroplets, Science 345, 906 (2014).

19. R. M. P. Tanyag, C. Bernando, C. F. Jones, C. Bacellar, K. R. Ferguson, D. Anielski, R. Boll, S. Carron, J. P. Cryan, L. Englert, S. Epp, B. Erk, L. Foucar, L. F. Gomez, R. Hartmann, D. M. Neumark, D. Rolles, B. Rudek, K. R. Siefermann, J. Ullrich, F. Weise, C. Bostedt, O. Gessner, and A. F. Vilesov, X-ray coherent diffraction imaging by immersion in nanodroplets, Structural Dynamics 2, 051102 (2015).

20. C. F. Jones, C. Bernando, R. M. P. Tanyag, C. Bacellar, K. R. Ferguson, L. F. Gomez, D. Anielski, A. Belkacem, R. Boll, J. Bozek, S. Carron, J. P. Cryan, L. Englert, S. W. Epp, B. Erk, L. Foucar, R. Hartmann, D. M. Neumark, D. Rolles, A. Rudenko, K. R. Siefermann, F. Weise, B. Rudek, F. P. Sturm, J. Ullrich, C. Bostedt, O. Gessner, and A. F. Vilesov, Coupled motion of Xe clusters and quantum vortices in He nanodroplets Phys. Rev. B. 93, 180510(R) (2016).

21. F. Ancilotto, M. Pi, and M. Barranco, Vortex arrays in nanoscopic superfluid helium droplets, Phys. Rev. B 91, 100503(R) (2015).

22. K. R. Ferguson, M. Bucher, J. D. Bozek, S. Carron, J.-C. Castagna, R. Coffee, G. I. Curiel, M. Holmes, J. Krzywinski, M. Messerschmidt, M. Minitti, A. Mitra, S. Moeller, P. Noonan, T. Osipov, S. Schorb, M. Swiggers, A. Wallace, J. Yina, and C. Bostedt, The atomic, molecular and optical science instrument at the linac coherent light source, J. Synchr. Rad. 22, 492 (2015).

23. M. Bucher, T. Osipov, J.-C. Castagna, S. Carron, K. Ferguson, M. Swiggers, A. Wallace, J. Aldrich, R. Armenta, G. G. Blaj, J. D. Bozek, R. Coffee, I. Curiel, R. Enert, L. Fang, C. Ford, L. Foucar, G. Gassner, P. A. Hart, R. Hartmann, G. Hauser, O. Hickmann, P. Holl, C. Kenney, J. Krzywinski, A. Mitra, D. Morton, P. Noonan, C. O'Grady, E. Paiser, J. Pines, E. Rodriguez, D. Rolles, A. Rudenko, R. Strecker, L. Struder, J. Thayer, M. Weaver, H. Xiong, J. Yin, D. Zhang, J. Ullrich, N. Berrah, and C. Bostedt, The LAMP endstation for soft x-ray imaging and spectroscopy experiments at the Atomic, Molecular, and Optical Science instrument of the Linac Coherent Light Source, (in preparation).

24. J. P. Toennies, and A. F. Vilesov, Superfluid helium droplets: A uniquely cold nanomatrix for molecules and molecular complexes, Angew. Chem. Int. Ed. 43, 2622 (2004).

25. M. Y. Choi, G. E. Douberly, T. M. Falconer, W. K. Lewis, C. M. Lindsay, J. M. Merritt, P. L. Stiles, and R. E. Miller, Infrared spectroscopy of helium nanodroplets: novel methods for physics and chemistry, Int. Rev. Phys. Chem. 25, 15 (2006). 
26. C. Callegari, and W. E. Ernst, Helium Droplets as Nanocryostats for Molecular Spectroscopyfrom the Vacuum Ultraviolet to the Microwave Regime, in Handbook of High-resolution Spectroscopy., edited by M. Quack and F. Merkt (John Wiley \& Sons, Ltd., 2011), pp. 1551.

27. M. Mudrich, and F. Stienkemeier, Photoionisaton of pure and doped helium nanodroplets, Int. Rev. Phys. Chem. 33, 301 (2014).

28. E. Loginov, L. F. Gomez, N. Chiang, A. Halder, N. Guggemos, V. V. Kresin, and A. F. Vilesov, Photoabsorption of $\mathrm{Ag}_{\mathrm{N}}$ ( $\left.\mathrm{N} \sim 6-6000\right)$ nanoclusters formed in helium droplets: $A$ transition from compact to multi-center aggregation, Phys. Rev. Lett. 106, 233401 (2011).

29. L. F. Gomez, E. Loginov, R. Sliter, and A. F. Vilesov, Sizes of large helium droplets, J. Chem. Phys. 135, 154201 (2011).

30. L. F. Gomez, E. Loginov, and A. F. Vilesov, Traces of vortices in superfluid helium droplets, Phys. Rev. Lett. 108, 155302 (2012).

31. L. Strüder, S. Epp, D. Rolles, R. Hartmann, P. Holl, G. Lutz, H. Soltau, R. Eckart, C. Reich, K. Heinzinger, C. Thamm, A. Rudenko, F. Krasniqi, K. U. Kuhnel, C. Bauer, C. D. Schroter, R. Moshammer, S. Techert, D. Miessner, M. Porro, O. Halker, N. Meidinger, N. Kimmel, R. Andritschke, F. Schopper, G. Weidenspointner, A. Ziegler, D. Pietschner, S. Herrmann, U. Pietsch, A. Walenta, W. Leitenberger, C. Bostedt, T. Moller, D. Rupp, M. Adolph, H. Graafsma, H. Hirsemann, K. Gartner, R. Richter, L. Foucar, R. L. Shoeman, I. Schlichting, and J. Ullrich, Largeformat, high-speed, X-ray pnCCDs combined with electron and ion imaging spectrometers in a multipurpose chamber for experiments at 4 th generation light sources, Nucl. Instrum. Meth. A 614, 483 (2010).

32. T. Gorkhover, M. Adolph, D. Rupp, S. Schorb, S. W. Epp, B. Erk, L. Foucar, R. Hartmann, N. Kimmel, K. U. Kühnel, D. Rolles, B. Rudek, A. Rudenko, R. Andritschke, A. Aquila, J. D. Bozek, N. Coppola, T. Erke, F. Filsinger, H. Gorke, H. Graafsma, L. Gumprecht, G. Hauser, S. Herrmann, H. Hirsemann, A. Hömke, P. Holl, C. Kaiser, F. Krasniqi, J. H. Meyer, M. Matysek, M. Messerschmidt, D. Miessner, B. Nilsson, D. Pietschner, G. Potdevin, C. Reich, G. Schaller, C. Schmidt, F. Schopper, C. D. Schroter, J. Schulz, H. Soltau, G. Weidenspointner, I. Schlichting, L. Strüder, J. Ullrich, T. Möller, and C. Bostedt, Nanoplasma dynamics of single large xenon clusters irradiated with superintense X-ray pulses from the linac coherent light source free-electron laser, Phys. Rev. Lett. 108, 245005 (2012).

33. C. Bostedt, J. D. Bozek, P. H. Bucksbaum, R. N. Coffee, J. B. Hastings, Z. Huang, R. Lee, W., S. Schorb, J. N. Corlett, P. Denes, P. Emma, R. W. Falcone, R. W. Schoenlein, G. Doumy, E. P. Kanter, B. Kraessig, S. Southworth, L. Young, L. Fang, M. Hoener, N. Berrah, C. Roedig, and L. F. DiMauro, Ultra-fast and ultra-intense $x$-ray sciences: first results from the Linac Coherent Light Source free-electron laser, J. Phys. B 46, 164003 (2013).

34. P. Roche, M. Roger, and F. I. B. Williams, Interpretation of the low damping of subthermal capillary waves (ripplons) on superfluid He-4, Phys. Rev. B 53, 2225 (1996).

35. H. Lamb, Hydrodynamics, 6 ed. (Dover, New York, 1945).

36. I. Barke, H. Hartmann, D. Rupp, L. Flukiger, M. Sauppe, M. Adolph, S. Schorb, C. Bostedt, R. Treusch, C. Peltz, S. Bartling, T. Fennel, K. H. Meiwes-Broer, and T. Moller, The 3D-architecture of individual free silver nanoparticles captured by X-ray scattering, Nat Commun 6, 7185 (2015).

37. C. Bostedt, H. N. Chapman, J. T. Costello, J. R. C. Lopez-Urrutia, S. Dusterer, S. W. Epp, J. Feldhaus, A. Fohlisch, M. Meyer, T. Moller, R. Moshammer, M. Richter, K. Sokolowski-Tinten, A. Sorokin, K. Tiedtke, J. Ullrich, and W. Wurth, Experiments at FLASH, Nucl Instrum Meth A 601, 108 (2009).

38. C. Svetina, C. Grazioli, N. Mahne, L. Raimondi, C. Fava, M. Zangrando, S. Gerusina, M. Alagia, L. Avaldi, G. Cautero, M. de Simone, M. Devetta, M. Di Fraia, M. Drabbels, V. Feyer, P. Finetti, R. Katzy, A. Kivimaki, V. Lyamayev, T. Mazza, A. Moise, T. Moller, P. O'Keeffe, Y. Ovcharenko, P. 
Piseri, O. Plekan, K. C. Prince, R. Sergo, F. Stienkemeier, S. Stranges, M. Coreno, and C. Callegari, The Low Density Matter (LDM) beamline at FERMI: optical layout and first commissioning, J Synchrotron Radiat 22, 538 (2015).

39. D. Rupp, N. Monserud, B. Langbehn, M. Sauppe, J. Zimmermann, O. Y., T. Moller, F. Frassetto, L. Poletto, A. Trabattoni, F. Calegari, M. Nisoli, K. Sander, C. Peltz, M. J. J. Vrakking, T. Fennel, and R. e. A., Single-shot coherent diffractive imaging of helium nanodroplets with a high-harmonic generation source, http://arxiv.org/abs/1610.05997 (2016).

40. V. B. Eltsov, R. de Graaf, P. J. Heikkinen, J. J. Hosio, R. Hanninen, and M. Krusius, Vortex Formation and Annihilation in Rotating Superfluid He-3-B at Low Temperatures, J. Low Temp. Phys. 161, 474 (2010).

41. R. Hanninen, and A. W. Baggaley, Vortex filament method as a tool for computational visualization of quantum turbulence, Proc. Natl. Acad. Sci. USA 111, 4667 (2014).

42. S. Yui, and M. Tsubota, Counterflow quantum turbulence of He-II in a square channel: Numerical analysis with nonuniform flows of the normal fluid, Phys. Rev. B 91, 184504 (2015).

43. J. R. Abo-Shaeer, C. Raman, J. M. Vogels, and W. Ketterle, Observation of vortex lattices in BoseEinstein condensates, Science 292, 476 (2001).

44. M. Hartmann, R. E. Miller, J. P. Toennies, and A. Vilesov, Rotationally resolved spectroscopy of $\mathrm{SF}_{6}$ in liquid-helium clusters - a molecular probe of cluster temperature, Phys. Rev. Lett. 75, 1566 (1995).

45. S. Grebenev, J. P. Toennies, and A. F. Vilesov, Superfluidity within a small helium-4 cluster: The microscopic Andronikashvili experiment, Science 279, 2083 (1998).

46. R. J. Donnelly, and C. F. Barenghi, The Observed Properties of Liquid Helium at the Saturated Vapor Pressure, J. Phys. Chem. Ref. Data 27, 1217 (1998).

47. W. H. Zurek, Cosmological Experiments in Superfluid-Helium, Nature 317, 505 (1985).

48. C. Bauerle, Y. M. Bunkov, S. N. Fisher, H. Godfrin, and G. R. Pickett, Laboratory simulation of cosmic string formation in the early Universe using superfluid He-3, Nature 382, 332 (1996).

49. Y. M. Bunkov, A. I. Golov, V. S. L'vov, A. Pomyalov, and I. Procaccia, Evolution of a neutroninitiated micro big bang in superfluid He-3-B, Phys. Rev. B 90, 024508 (2014). 